\title{
Mechanism of age-dependent susceptibility and novel treatment strategy in glutaric acidemia type I
}

\author{
William J. Zinnanti, ${ }^{1}$ Jelena Lazovic, ${ }^{2}$ Cathy Housman, ${ }^{3}$ Kathryn LaNoue, ${ }^{4}$ James P. O'Callaghan, ${ }^{5}$ \\ Ian Simpson, ${ }^{6}$ Michael Woontner, ${ }^{7}$ Stephen I. Goodman, ${ }^{7}$ James R. Connor, ${ }^{8}$ \\ Russell E. Jacobs, ${ }^{2}$ and Keith C. Cheng 1
}

\begin{abstract}
1Jake Gittlen Cancer Research Foundation, Department of Pathology, Department of Biochemistry \& Molecular Biology, and Department of Pharmacology, Penn State College of Medicine, Hershey, Pennsylvania, USA. 'Beckman Institute, California Institute of Technology, Pasadena, California, USA.

${ }^{3}$ Department of Pathology and 4Department of Cellular \& Molecular Physiology and Department of Biochemistry, Penn State College of Medicine, Hershey, Pennsylvania, USA. ${ }^{5}$ Centers for Disease Control and Prevention, National Institute for Occupational Safety and Health, Morgantown, West Virginia, USA. ${ }^{6}$ Department of Neural and Behavioral Sciences, Penn State College of Medicine, Hershey, Pennsylvania, USA. 'Department of Pediatrics, University of Colorado at Denver and Health Sciences Center, Denver, Colorado, USA. ${ }^{8}$ Department of Neurosurgery,
\end{abstract}

Penn State College of Medicine, Hershey, Pennsylvania, USA.

\begin{abstract}
Glutaric acidemia type I (GA-I) is an inherited disorder of lysine and tryptophan metabolism presenting with striatal lesions anatomically and symptomatically similar to Huntington disease. Affected children commonly suffer acute brain injury in the context of a catabolic state associated with nonspecific illness. The mechanisms underlying injury and age-dependent susceptibility have been unknown, and lack of a diagnostic marker heralding brain injury has impeded intervention efforts. Using a mouse model of GA-I, we show that pathologic events began in the neuronal compartment while enhanced lysine accumulation in the immature brain allowed increased glutaric acid production resulting in age-dependent injury. Glutamate and GABA depletion correlated with brain glutaric acid accumulation and could be monitored in vivo by proton nuclear magnetic resonance ( ${ }^{1} \mathrm{H}$ NMR) spectroscopy as a diagnostic marker. Blocking brain lysine uptake reduced glutaric acid levels and brain injury. These findings provide what we believe are new monitoring and treatment strategies that may translate for use in human GA-I.
\end{abstract}

\section{Introduction}

Glutaryl-CoA dehydrogenase (EC 1.3.99.7) (GCDH) activity is required for complete oxidation of lysine and tryptophan. Autosomal recessive inheritance of GCDH deficiency, known as glutaric acidemia type I (GA-I), is one of the more common inherited metabolic disorders affecting 1 out of 30,000-100,000 children worldwide $(1,2)$. GA-I results in accumulation of glutaric acid and glutarylcarnitine with carnitine deficiency (3-5). Affected individuals accumulate substantial glutaric acid in the brain and 3-hydroxyglutaric acid (3-OHGA) to a lesser extent (6-8). Following a period of normal development, affected children may experience irreversible striatal injury during an encephalopathic crisis, commonly precipitated by a nonspecific illness between 6 and 18 months of age (2). The neuropathology associated with GA-I is similar to that of Huntington disease, in which a systemic genetic defect results in specific striatal degeneration involving loss of medium spiny neurons and astrogliosis (6-10).

Current therapy for GA-I includes dietary lysine restriction, carnitine supplementation, and emergency treatment of intercurrent illness including intravenous glucose (3-5). Unfortunately, one-third of affected children, prospectively identified by new-

Nonstandard abbreviations used: BBB, blood-brain barrier; ChAT, choline acetyltransferase; Cr, creatine; GA-I, glutaric acidemia type I; GCDH, glutaryl-CoA dehydrogenase; GFAP, glial fibrillary acidic protein; Glx, glutamate and glutamine; ${ }^{1} \mathrm{H}$ NMR, proton nuclear magnetic resonance; NAA, N-acetyl aspartate; NeuN, neuron-specific nuclear protein; Occl, occludin; 3-OHGA, 3-hydroxyglutaric acid; PCA, perchloric acid; $\mathrm{T}_{2}$, transverse relaxation time constant.

Conflict of interest: The authors have declared that no conflict of interest exists. Citation for this article: J. Clin. Invest. 117:3258-3270 (2007). doi:10.1172/JCI31617. born screening, do not respond to therapy and experience striatal degeneration despite careful clinical management (11). Children with GA-I who escape encephalopathy in their first 5 years typically remain asymptomatic (2). We recently developed an animal model of GA-I encephalopathy in GCDH-deficient $\left(\mathrm{Gcdh}^{-/-}\right)$mice by introducing added dietary protein or lysine (12). This model shows age-dependent susceptibility to acute brain injury similar to human GA-I. Weanling (4-week-old) $\mathrm{Gcdh}^{-/-}$mice accumulate substantial glutaric acid and suffer seizures, paralysis, hemorrhages, and death within 3-6 days of lysine diet exposure. In contrast, adult (8-week-old) $\mathrm{Gcdh}^{-/-}$mice survive lysine exposure and accumulate less glutaric acid, though all develop bilateral striatal lesions after 6 weeks of lysine diet exposure.

In the current study, we first localized the origin of brain injury by identifying the cell type or types in the brain that express Gcdh and the earliest pathological events after protein and lysine supplementation in weanling $G c d b^{-/-}$mice. We then compared the biochemical responses in weanling and adult $\mathrm{Gcdh}^{-/-}$mice after lysine diet challenge to identify differences associated with age-dependent susceptibility. Our current findings identified developmental differences that control glutaric acid accumulation and age-dependent brain injury. Brain injury was preceded by biochemical changes that may be used as a diagnostic marker. These findings led to what we believe are novel monitoring and treatment strategies.

\section{Results}

Expression pattern of Gcdh predicts neuronal origin of pathologic events in the brain. To better understand the mechanism of brain injury, we 

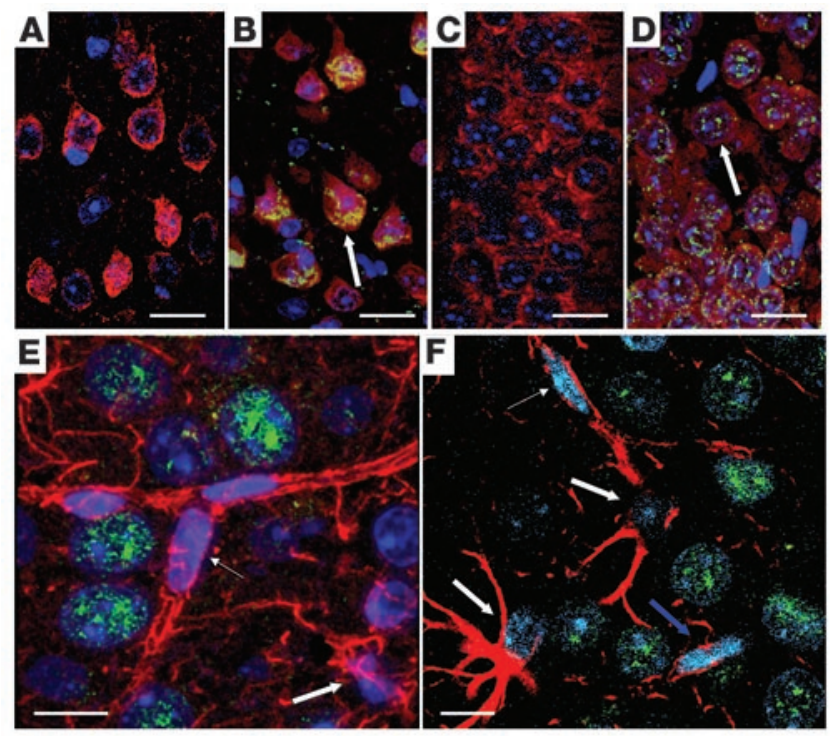

\section{Figure 1}

Neuronal localization of Gcdh expression ( $\beta$-gal). Motor cortex of WT (A) and $\mathrm{Gcdh}^{-/+}$mouse (B) shows positive $\beta$-gal (green) staining colocalizes (yellow) with neurons (red) in layer $\mathrm{V}$ and $\mathrm{VI}$ of $\mathrm{Gcdh} h^{-/+}$mouse and not WT by immunofluorescence confocal microscopy. CA1 hippocampus from WT (C) and $\mathrm{Gcdh}^{-/+}$mouse (D) shows positive $\beta$-gal staining for $\mathrm{Gcdh}^{-/+}$mouse neurons only, similar to that found in motor cortex. Arrows indicate color change. (E) Average projection of 10 confocal slices through the CA1 hippocampus of a $\mathrm{Gcdh}^{-/+}$mouse shows no $\beta$-gal staining in endothelial cells (thin arrow) or astrocytes (red) (thick arrows). (F) A single slice through the CA1 hippocampus shows that nuclei associated with astrocytes (thick arrows) and endothelial cells (thin arrow) do not stain positive for $\beta$-gal while large nuclei of neurons are positively stained. $\beta$-Gal labeling is nuclear due to nuclear localizing sequence; see Methods. (A-D) $\beta$-Gal, green; NeuN, red; colocalization $\beta$-gal and NeuN, yellow; and DAPI, blue. Scale bars: $10 \mu \mathrm{m}$. (E and F) $\beta$-Gal, green; GFAP, red; and DAPI, blue. Scale bars: $10 \mu \mathrm{m}$. used double-label immunohistochemistry to determine whether $G c d h$ expression is limited to neurons, astrocytes, or endothelial cells. We then used electron and confocal microscopy to detect the earliest pathologic events in the brain after special diet exposure. Sections of motor cortex and CA1 hippocampus from $\mathrm{Gcdh} h^{-/+}$mice revealed that $G c d h$ expression was limited to neurons (indicated by $\beta$-gal labeling; see Methods) (Figure 1, A-F).

Pathologic changes were limited to $G c d h^{-/-}$mice and were evident within 24-48 hours of protein or lysine diet exposure in weanlings (Figure 2, A-H). Moderately vacuolated neurons were found in the motor cortex with pathological changes previously associated with necrosis (13) (Figure 2E). Dendritic processes in the motor cortex showed moderately enlarged mitochondria and disorganization of neurofilaments (Figure 2F). Swollen and disintegrating mitochondria were found in postsynaptic regions with edema of dendritic spines (Figure 2G), resembling excitotoxic lesions (9). Similar changes, though less severe, were found in adult $\mathrm{Gcdb^{-1- }}$ mice after 60 hours (Figures $2 \mathrm{H}$ and $3 \mathrm{~B}$ ). Although adult $G c d b^{-/-}$mice do not show obvious behavioral differences (12), further testing may reveal deficits associated with these pathologic changes after long-term diet exposure.

To confirm the cell types associated with vacuoles in a larger field, we compared confocal images of neurofilament- and astrocyte-labeled sections with semithin sections prepared for electron microscopy (Figure 3, A-D). The vacuoles that

\section{Figure 2}

formed in and around the pyramidal cell layer of CA3 hippocampus (Figure 3B) were surrounded by neurofilaments (shown by N52 labeling), indicating a neuronal origin of the vacuoles (Figure 3D). Vacuolation of astrocytes (glial fibrillary acidic protein [GFAP]) was not apparent. Additionally, labeling of cholinergic neurons showed vacuole formation in the cell bodies and processes of these large neurons of the striatum within 48 hours of lysine
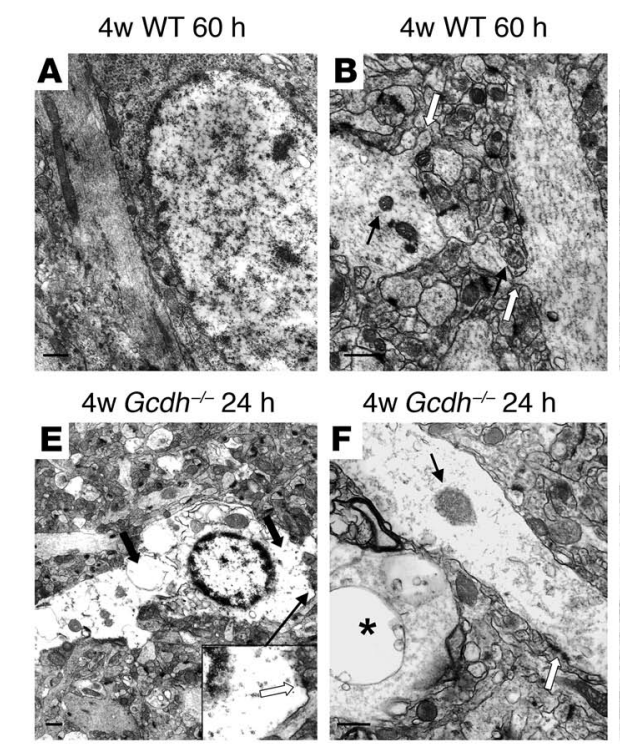

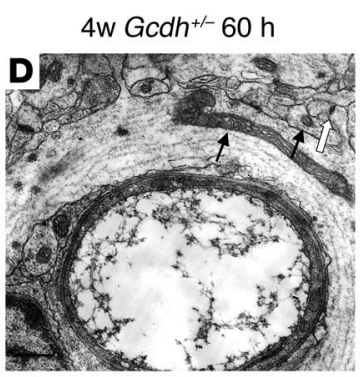

8 w Gcdh $^{-/} 24 \mathrm{~h}$
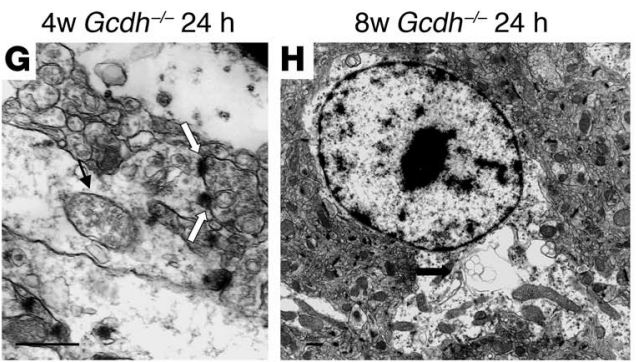

Ultrastructural changes after special diet exposure in $G c d h^{-1-}$ mice. Electron micrographs of motor cortex from weanling (4w) WT (A and B) and $\mathrm{Gcdh}^{-/+}(\mathbf{C}$ and $\mathbf{D})$ mice after 60 hours of protein diet show normal appearance including dendritic spines (white arrows) and mitochondria (black arrows). (E-G) Similar sections from weanling $\mathrm{Gcdh}^{-/-}$mouse motor cortex after 24 hours of protein diet shows vacuolated neuron ( $E$, thick arrows) identified by synapse ( $E$, thin arrow, inset) and peripheral chromatin aggregation in the nucleus. (F) A large vacuole is noted $\left(^{*}\right)$ adjacent to an edematous dendrite, identified by synapse (white arrow) with disintegrating mitochondrion (black arrow). (G) Higher magnification of section similar to that in $\mathbf{F}$ shows postsynaptic (white arrows) swelling with enlarged and disrupted mitochondrion (black arrow). (H) Similar section from adult (8w) $\mathrm{Gcdh}^{-/-}$mouse cortex shows vacuole formation after 60 hours of protein diet exposure. Scale bars: $1 \mu \mathrm{m}$. 


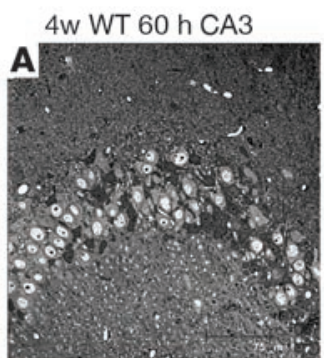

4w WT 60 h Striatum
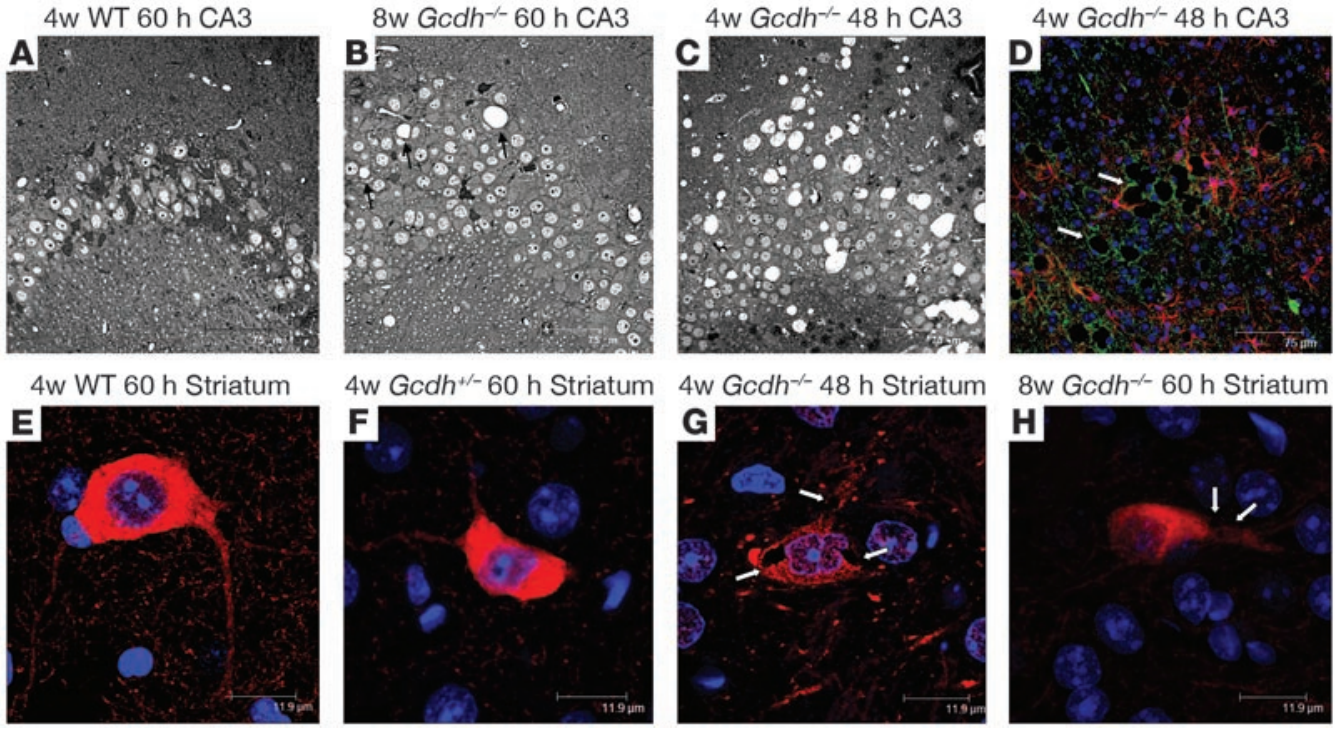

4w Gcdh ${ }^{-1-} 48$ h Striatum
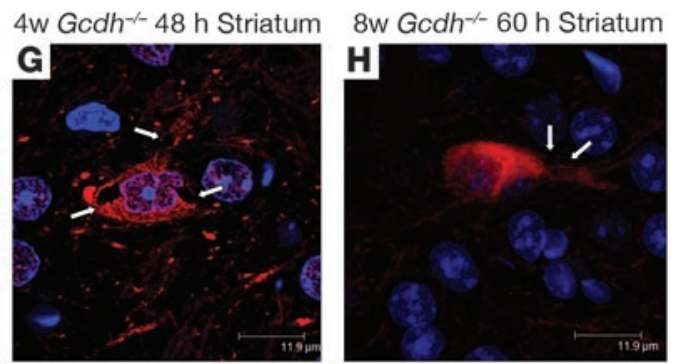

\section{Figure 3}

Neuronal vacuolation after special diet exposure in Gcdh-/- mice. (A) Semi-thin section of WT mouse CA3 hippocampus after 60 hours of protein diet shows normal appearance. (B) Similar section of adult Gcdh ${ }^{-/-}$mouse after 60 hours of protein diet shows a few vacuoles (black arrows). (C) A similar section from a weanling Gcdh ${ }^{-/}$ mouse after 48 hours of protein diet shows multiple vacuoles. (D) Confocal image of a section similar to that shown in $\mathbf{C}$ labeled with N52, GFAP, and DAPI shows vacuoles surrounded by N52 signal indicating neurofilaments (white arrows). (E-H) Acetylcholine transferase-positive neurons in the striatum of a weanling WT mouse (E) and weanling $\mathrm{Gcdh}^{-/+}$mouse (F) after 60 hours of lysine diet exposure show normal appearance. Development of vacuoles is prominent within the perikaryon and neuronal processes of a weanling $\mathrm{Gcd}^{-/-}$mouse (white arrows) after 48 hours of lysine diet exposure $(\mathbf{G})$ and less prominent in adult $G c d h^{-/}$mouse after 60 hours $(\mathbf{H})$. (D) N52, green; GFAP, red; and DAPI, blue. (E-H) ChAT, red; and DAPI, blue. 4w Gcdh ${ }^{+/} 60$ h Striatum

$(P<0.01)$ after 48 hours of lysine diet exposure (Figure 4A). Between 48 and 60 hours, a decrease in brain lysine was accompanied by an equimolar increase in glutaric acid, suggesting production of glutaric acid within the brain from available lysine (Figure 4A). At 8 weeks of age, adult $\mathrm{Gcdh}^{-/-}$mice showed no increase in brain lysine or glutaric acid levels on the lysine diet, consistent with a substantial reduction in brain lysine uptake with maturity. Changes in brain 3-OHGA paralleled those of glutaric acid although 3-OHGA concentrations were 20 -fold lower than glutaric acid levels (not shown). Although symptoms of encephalopathy were less evident at the earlier time points examined in the current work, brain glutaric acid levels correlate with acute injury of symptomatic mice as previously shown (12).

diet exposure in weanling $\mathrm{Gcdb^{-/- }}$ mice (Figure 3G). These changes were less noticeable in adults after 60 hours (Figure $3 \mathrm{H}$ ).

Brain lysine accumulation and age-dependent susceptibility. To determine the mechanism of age-dependent susceptibility to encephalopathy in Gcdh deficiency, we considered that brain glutaric acid levels were previously shown to correlate with severity of disease presentation (12). Therefore, we considered the possibilities that (a) brain lysine catabolism, which produces glutaric acid, may be reduced with age; (b) brain lysine uptake may be reduced with age; or (c) clearance of glutaric acid from the brain may be enhanced with age. Based on recent findings that showed limited bloodbrain barrier (BBB) permeability for glutaric acid (14), we pursued the first 2 possibilities.

We first tested for differences in brain lysine and glutaric acid accumulation between susceptible weanling $G c d b^{-1-}$ mice and encephalopathy-resistant adults. Since there were no significant differences between WT and heterozygotes with and without lysine diet exposure, we report these changes compared with heterozygous controls. All mice accumulated similar serum lysine levels with lysine diet exposure $(P<0.01)$ regardless of age or genotype (Figure $4 \mathrm{~A})$. Brain lysine levels were $46 \%$ higher in weanling $\mathrm{Gcdh}^{-/-}$mice compared with heterozygous controls, both of which were on a normal diet $(P<0.01)$ consistent with disrupted lysine catabolism in $\mathrm{Gcd} \mathrm{h}^{-/-}$mice (Figure 4A). Lysine diet exposure raised brain lysine levels by $66 \%$ in weanling heterozygotes $(P<0.01)$ without any increase in glutaric acid levels (Figure $4 \mathrm{~A})$. Weanling but not adult $\mathrm{Gcdb^{-/- }}$ mice showed a 3-fold increase in brain lysine accumulation $(P<0.001)$ accompanied by a 2 -fold increase in glutaric acid
To determine whether lysine diet exposure had any effect on other basic or neutral amino acids, we compared serum and brain alanine and arginine levels. Both serum arginine and alanine levels were 2-fold higher only in weanling $G c d h^{-/-}$mice on the lysine $\operatorname{diet}(P<0.01)$ (Figure 4B). On a normal diet, brain arginine levels were 2 -fold higher in weanling $G c d b^{-/-}$mice compared with heterozygotes $(P<0.01)$ (Figure 4B). Lysine diet exposure raised brain arginine $60 \%$ in both heterozygotes and weanling $\mathrm{Gcdh}^{-/-}$mice but not adults $(P<0.01$ and $P<0.001$ respectively, compared with heterozygote normal diet controls) (Figure 4B). Brain alanine levels were unaffected by lysine diet exposure in all groups (Figure 4B). These data are consistent with increased flux of the basic amino acid transporter in the immature brain that carries both lysine and arginine (15). that the lysine diet may induce catabolic stress in weanlings (16). Therefore, we measured serum glucose levels and $\beta$-hydroxybutyrate as an indicator of serum ketone bodies. Weanling $\mathrm{Gcdh}^{-/-}$mice on a normal diet had $27 \%$ lower serum glucose levels compared with heterozygotes $(P<0.01)$, which were reduced to less than half with lysine diet exposure $(P<0.001)$ (Figure $4 \mathrm{C})$. Adult $\mathrm{Gcdh^{-/- }}$ mice also had reduced serum glucose levels with lysine diet exposure compared with heterozygotes $(P<0.01)$ (Figure 4C). Serum $\beta$-hydroxybutyrate levels were $30 \%$ lower in weanling $\mathrm{Gcdh}^{-/-}$mice although lysine diet exposure raised $\beta$-hydroxybutyrate levels almost 4-fold in weanling $\mathrm{Gcdh}^{-/-}$mice and only about $40 \%$ in weanling heterozygotes $(P<0.001$ and $P<0.01$ respectively) (Fig-
Serum amino acid accumulation, other than lysine, suggested on a normal diet compared with heterozygous controls $(P<0.01)$ 

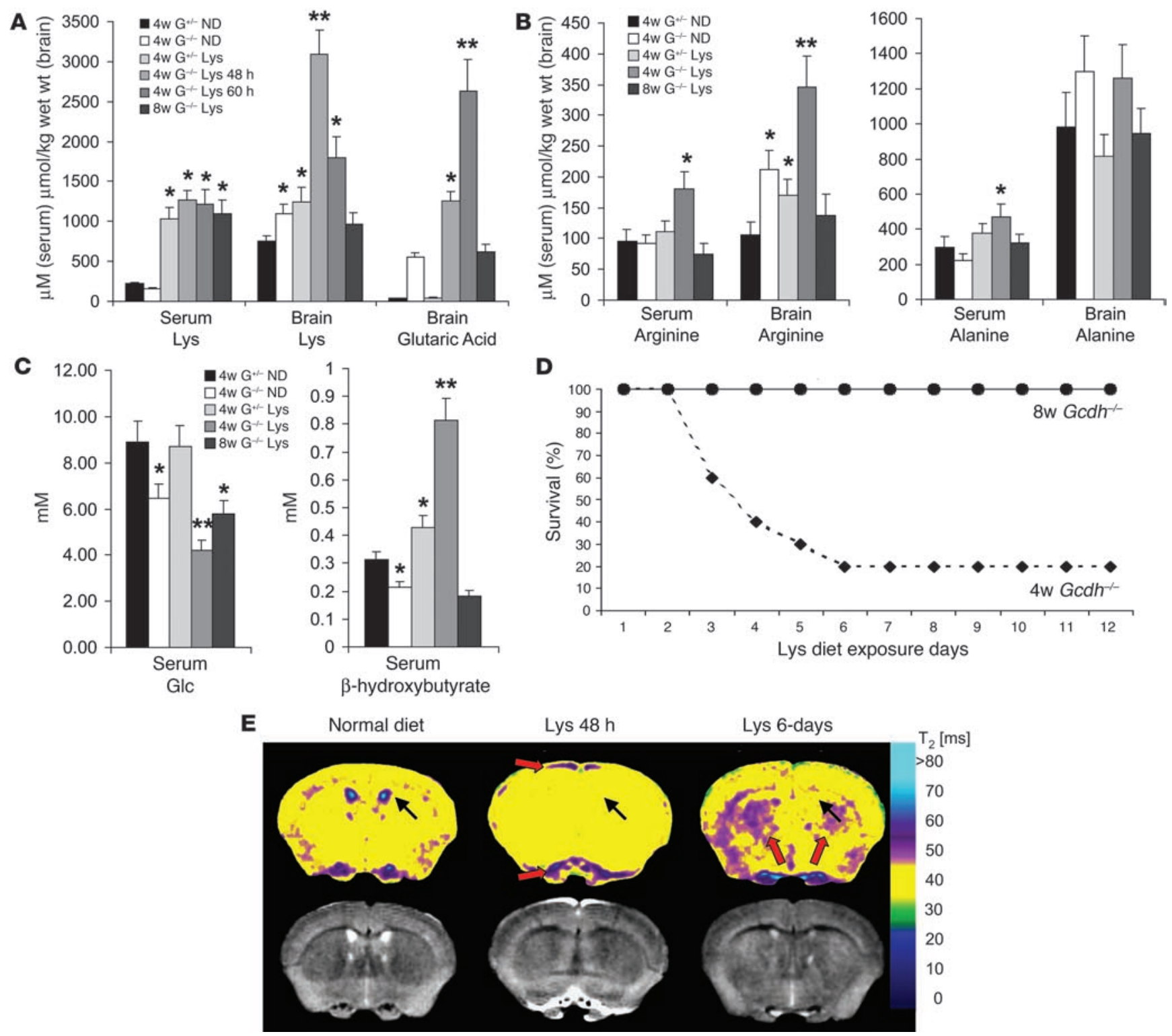

\section{Figure 4}

Brain lysine accumulation, ketotic hypoglycemia, and increased glutaric acid levels correlate with age-dependent susceptibility to brain injury in weanling Gcdh ${ }^{-/-}$mice. (A) Serum lysine, brain lysine, and brain glutaric acid levels, (B) serum and brain arginine and alanine levels, and (C) serum glucose (Glc) and $\beta$-hydroxybutyrate levels in weanling (4w) and adult (8w) Gcd $h^{-/-}$mice $\left(\mathrm{G}^{-/-}\right)$and heterozygous controls $\left(\mathrm{G}^{-/+}\right)$on normal (ND) or lysine (Lys) diets. Amino acid differences are compared with heterozygous normal diet controls, and brain glutaric acid differences are compared with weanling Gcd $h^{-/}$normal diet controls. Mean \pm SEM, ${ }^{*} P<0.01$; ${ }^{* *} P<0.001 . n=6$ each group. (D) Twelve-day survival of adult (black circles, $n=20$ ) and weanling (black diamonds, $n=20) \mathrm{Gcd} h^{-/-}$mice on the lysine diet. (E) $\mathrm{T}_{2}$ maps (top) and $\mathrm{T}_{2}$-weighted images (bottom) of weanling $\mathrm{Gcdh}^{-/}$mice. Color bar indicates $\mathrm{T}_{2}$ values (right side). Weanlings showed brain swelling, indicated by obliterated ventricles (black arrows; compare left with middle and right), and increased subdural fluid collection (red arrows, middle) at 48 hours of lysine diet exposure. At 6 days, surviving weanlings developed striatal lesions indicated by increased $\mathrm{T}_{2}$ signal (red arrows, right).

ure 4C). These changes are consistent with induction of catabolic stress after lysine diet exposure. Additionally, the reduced serum glucose with increased serum alanine levels may represent an impairment of gluconeogenesis (16).

Increased brain glutaric acid accumulation in weanling $\mathrm{Gcdh} \mathrm{h}^{-/}$ mice was associated with $20 \% 12$-day survival compared with $100 \%$ survival of adults on the lysine diet (Figure 4D). Weanlings showed brain swelling by MRI at 48 hours and development of bilateral striatal lesions at 6 days on the lysine diet (Figure 4E). Together, these data indicate that enhanced lysine uptake in the immature brain provides increased glutaric acid accumulation and susceptibility to brain injury.

Brain biochemical changes consistent with disrupted mitochondrial function. Mitochondrial swelling and disruption in the brain of $\mathrm{Gcdh}^{-/-}$ mice indicate disrupted mitochondrial function with lysine diet exposure. Gcdh deficiency may lead to the intramitochondrial 

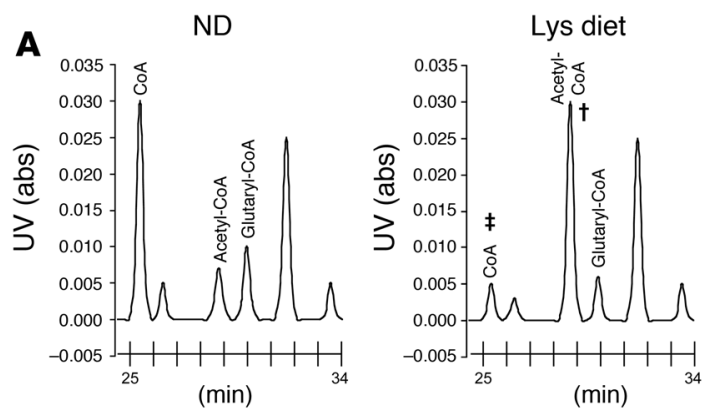

B
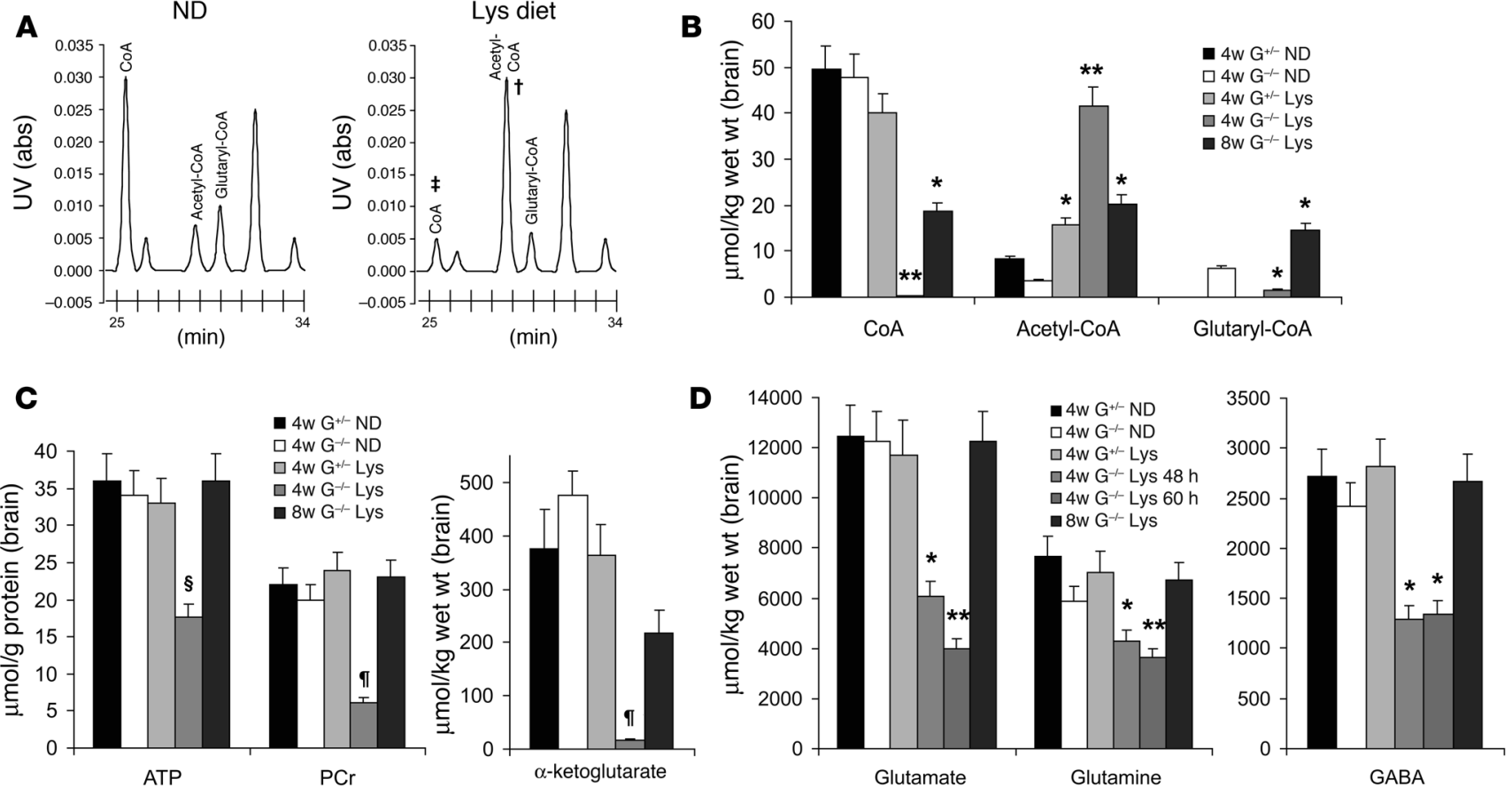

Figure 5

Age-dependent brain biochemical changes with lysine diet exposure. (A) Chromatogram of acyl-CoA esters from brain extract of weanling $\mathrm{Gcdh}^{-/}$mice on normal or lysine diet shows large accumulation of acetyl-CoA ( $\dagger$ ) and depletion of free CoA ( $\left.¥\right)$ after 48 hours of lysine diet. Abs, absorbance. (B) Free CoA, acetyl-CoA, and glutaryl-CoA changes in brain extract of weanling and adult Gcd $h^{-/-}$mice and heterozygous controls on normal or lysine diets. Mean \pm SEM, ${ }^{*} P<0.01 ;{ }^{* \star} P<0.001 . n=6$ each group. (C) ATP, phosphocreatine (PCr), and $\alpha-k e t o g l u t a r a t e$ changes in cortex of weanling and adult $\mathrm{Gcdh}^{-/}$mice and heterozygous controls on the lysine diet compared with normal diet. Mean \pm SEM, $\S P<0.04$; $\uparrow P<0.02$. $n=4$ each group. (D) Glutamate, glutamine, and GABA levels in weanling and adult Gcd $h^{-/-}$mice and heterozygous controls on the lysine diet compared with normal diet. Mean \pm SEM. $n=6$ each group.

accumulation of glutaryl-CoA, which may result in sequestration of intramitochondrial free CoA. Alternatively, increased ketone body utilization leads to acetyl-CoA accumulation, which can also reduce free CoA levels $(17,18)$. Therefore, we measured acetyl-CoA, glutaryl-CoA, free CoA, ATP, phosphocreatine, and $\alpha$-ketoglutarate in the brain of weanling and adult $\mathrm{Gcdh} b^{-/-}$mice or heterozygote controls with normal and lysine diet exposure (Figure 5, A-D). Heterozygote controls on the lysine diet did not accumulate glutaryl-CoA but did show a 2 -fold increase in acetyl-CoA $(P<0.01)$, indicating increased ketone body utilization consistent with the increased serum $\beta$-hydroxybutyrate levels in this group (Figure 5B). Weanling $G c d b^{-/-}$mice showed severe depletion of free CoA to $0.6 \%$ of normal $(P<0.001)$ on the lysine diet. However, glutaryl-CoA was unexpectedly reduced $(P<0.02)$, and acetyl-CoA was shown to accumulate to more than 10 -fold normal diet levels $(P<0.02)$ (Figure 5, A and B). On the other hand, adult $\mathrm{Gcdh}^{-/-}$mice showed a 2-fold increase in glutaryl-CoA $(P<0.02)$ and a 5 -fold increase in acetyl-CoA $(P<0.05)$ with depletion of free CoA to $38 \%$ of normal $(P<0.001)$ on the lysine diet (Figure 5B). Cortical ATP $(P<0.04)$ and phosphocreatine $(P<0.02)$ levels were depleted with lysine diet exposure only in weanling $\mathrm{Gcd} b^{-/-}$mice (Figure $5 \mathrm{C}$ ). Whole-brain $\alpha$-ketoglutarate levels were depleted to $3 \%$ of normal $(P<0.001)$ in weanling $G c d b^{-/-}$mice on the lysine diet, consistent with reduced ATP levels and Krebs cycle disruption (Figure 5C). Adult $\mathrm{Gcdh}^{-/-}$mice showed $\alpha$-ketoglutarate levels reduced to $46 \%$ of normal $(P<0.01)$ but no significant deficit in ATP or phosphocreatine (Figure 5C).
Increased ketone body utilization was previously shown to sequester free CoA and cause accumulation of $\alpha$-ketoglutarate, since free $\mathrm{CoA}$ is a limiting substrate for $\alpha$-ketoglutarate dehydrogenase (17). Depletion of $\alpha$-ketoglutarate with acetyl-CoA accumulation in $G c d b^{-/-}$mice suggests a different mechanism, involving glutaric acid accumulation and resulting in CoA sequestration. We propose that loss of Krebs cycle intermediates through $\alpha$-ketoglutarate may be a primary cause of mitochondrial disruption as diagrammed in Figure 6. Accumulation of saccharopine, previously reported in human GA-I (8), may sequester $\alpha$-ketoglutarate directly. Alternatively, glutaric acid accumulation may deplete Krebs cycle intermediates through exchange with $\alpha$-ketoglutarate (Figure 6). Loss of Krebs cycle intermediates would prevent the regeneration of oxaloacetate, which is needed to combine with acetyl-CoA to form citrate for continued Krebs cycle function. The resulting accumulation of acetyl-CoA depletes available free $\mathrm{CoA}$ and depresses the buildup of glutaryl-CoA due to the limited mitochondrial CoA pool.

Since $\alpha$-ketoglutarate is a precursor of glutamate synthesis (19) and the product of glutamate dehydration (20), depletion of $\alpha$-ketoglutarate suggests that glutamate may be depleted as well. Additionally, GABA is produced from glutamate decarboxylation and was previously shown to be depleted in human GA-I (21). Therefore we compared glutamate, glutamine, and GABA levels in the brain of weanling $G c d b^{-/-}$mice with and without lysine diet exposure (Figure 5D). Glutamate and GABA levels were depleted 


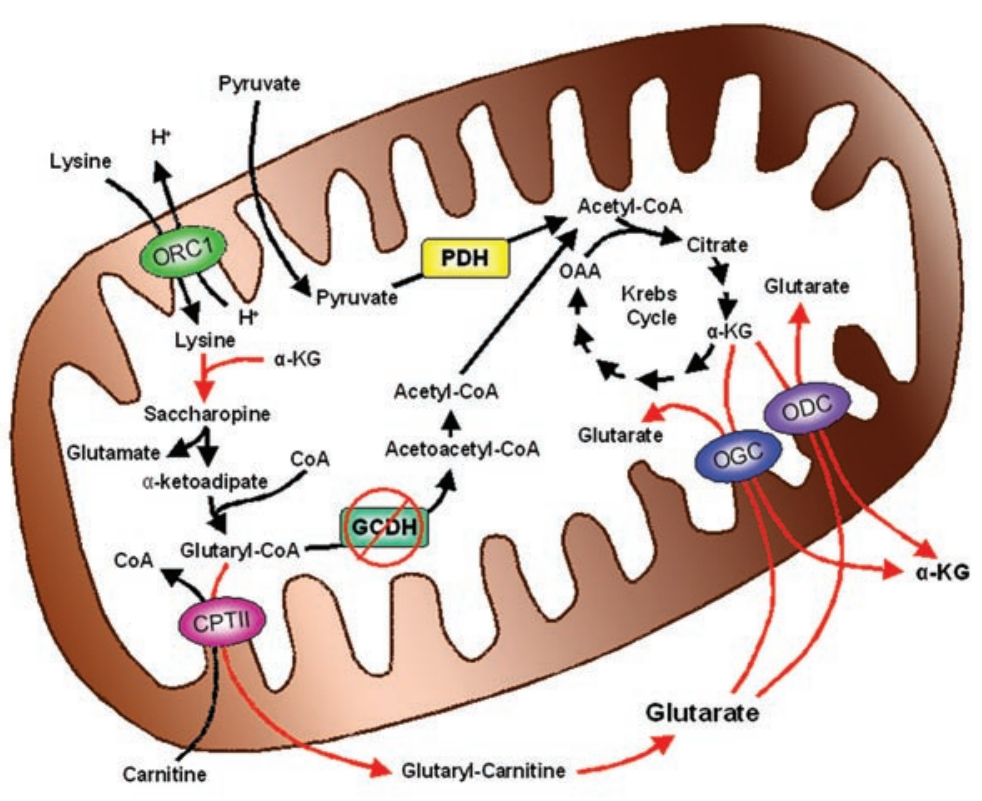

\section{Figure 6}

Proposed mechanism of mitochondrial dysfunction. Normal mitochondrial metabolism involves lysine uptake through the ornithine carrier (ORC1) with proton exchange. Lysine degradation is initiated by combination with $\alpha$-ketoglutarate $(\alpha-K G)$ to form saccharopine. Dehydrogenation of saccharopine forms glutamate and aminoadipic semialdehyde, which is further oxidized to $\alpha$-ketoadipate. Glutaryl-CoA is then formed from oxidation of $\alpha$-ketoadipate with addition of free $\mathrm{CoA}$. GCDH is required for complete oxidation with the formation of acetyl-CoA that can enter the Krebs cycle. GCDH deficiency results in disruption of normal lysine breakdown, which may cause saccharopine to accumulate and sequester $\alpha-K G$. Alternatively, accumulating free glutaric acid (glutarate) may deplete $\alpha$-KG levels by a strict counter-exchange mechanism via the oxodicarboxylate carrier (ODC) and to a lesser extent through the oxoglutarate carrier (OGC) that normally functions in the malate/aspartate shuttle. Depletion of $\alpha-K G$ prevents regeneration of oxaloacetate (OAA) needed to combine with acetyl-CoA to form citrate for continued Krebs cycle function. Acetyl-CoA is unable to enter the Krebs cycle and accumulates. CPTII, carnitine palmitoyltransferase II; $\mathrm{PDH}$, pyruvate dehydrogenase. to half the normal concentrations $(P<0.001)$ after lysine diet exposure while glutamine levels were reduced by only $24 \%(P<0.01)$ (Figure 5D). Since glutamine is exclusively produced in glia (22), depletion of more glutamate than glutamine in this model suggests a neuronal origin of metabolic impairment. GABA depletion may be responsible for the seizures observed in children and mice with Gcdh deficiency $(7,12)$.

Limiting brain lysine uptake and catabolism provides successful treatment. The increase in brain lysine and glutaric acid associated with susceptibility in weanling $G c d b^{-/-}$mice suggested the possibility that (a) blocking brain lysine uptake or (b) reducing brain lysine catabolism may be protective. We tested the first possibility by administration of homoarginine, previously shown to effectively compete with brain lysine uptake (23). We tested the second possibility by administration of glucose to reduce lysine catabolism. We administered these treatments separately or combined to weanling $\mathrm{Gcd} \mathrm{b}^{-/-}$mice on the lysine diet and monitored these animals for survival, biochemical changes, and brain injury by MRI (Figures 7 and 8). Each treatment substantially reduced brain glutaric acid accumulation, improved survival, and prevented brain injury in surviving animals at 6 days (Figure 7) although mildly increased transverse relaxation time constant $\left(T_{2}\right)$ values, representing vasogenic edema (24), were found in the striatum of some glucose-only treated mice (Figure 7F). Glucose treatment reduced accumulation of brain lysine by one-third $(P<0.03)$ and glutaric acid by $45 \%(P<0.001)$, resulting in a 3 -fold increase in 12 -day survival (Figure 7, A and D). Homoarginine treatment reduced brain lysine accumulation by $50 \%(P<0.01)$, resulting in $59 \%$ less glutaric acid accumulation $(P<0.001)$ and a 4 -fold increase in 12-day survival (Figure 7, B and D). Combined homoarginine/ glucose treatment provided similar control of lysine and glutaric acid compared with homoarginine alone but improved 12-day survival to $100 \%$ (Figure $7, \mathrm{C}$ and D).

Treatment success correlated directly with control of brain glutaric acid levels and maintenance of glutamate and GABA. Glutamate and GABA levels were higher with glucose treatment compared with lysine diet controls (Figure 8A). However, homoar- ginine alone and combined treatment maintained glutamate and GABA levels closest to normal (Figure 8A). Glutamine levels were further reduced with each treatment $(P<0.01)$ (Figure 8A). Glucose and homoarginine treatment each protected free CoA, glutaryl-CoA, and $\alpha$-ketoglutarate levels $(P<0.01)$ although only combined treatment decreased acetyl-CoA accumulation $(P<0.01)$, which resulted in substantially higher levels of free CoA compared with either treatment alone (Figure 8B). These effects are consistent with protection of Krebs cycle function.

The protection of free CoA with combined treatment suggested that carnitine supplementation, routinely used to treat human GA-I (2), may be protective in this mouse model. However, carnitine supplementation at up to 4 times the level given to children during encephalopathic crisis (100 mg/kg every 6 hours) (5) did not provide any improvement in survival. The lack of benefit from carnitine in this model may be related to limited access of dietary supplementation $(25,26)$ or a limited ability to conjugate glutarate in the brain as previously suggested (14).

Glutamate, glutamine, and GABA levels measured by ${ }^{1} H \mathrm{NMR}$ spectroscopy provide a diagnostic marker. Serum glutaric acid levels do not correlate with risk or progression of brain injury in human GA-I (11). Our current and previous data with this mouse model indicate that brain glutaric acid levels correlate with injury (12). However, brain glutaric acid levels currently cannot be monitored noninvasively and a clinical marker is needed to help guide treatment efforts (27). Glutamate and GABA depletion are shown here to correlate with elevated brain glutaric acid levels and progression of injury. The magnitude of glutamate and GABA depletion detected in brain extracts suggested that this biochemical change may be detectable by proton nuclear magnetic resonance $\left({ }^{1} \mathrm{H}\right.$ NMR) spectroscopy and used as a diagnostic marker. Therefore, we performed ${ }^{1} \mathrm{H}$ NMR spectroscopy and MRI to correlate progression of encephalopathy with glutamate and GABA levels (Figure 9). Weanling $G c d h^{-/-}$mice showed worsening symptoms associated with progression of encephalopathy between 48 and 60 hours of lysine diet exposure. A substantial reduction in the glutamate and 

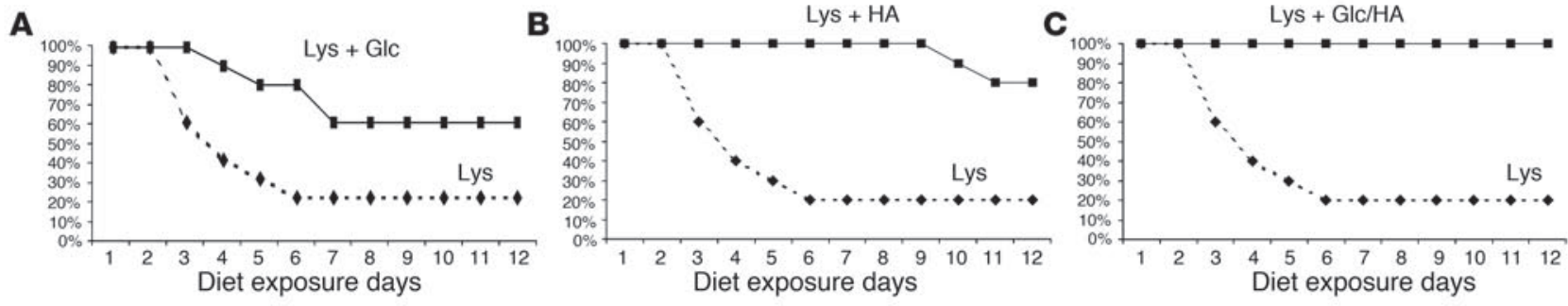

D
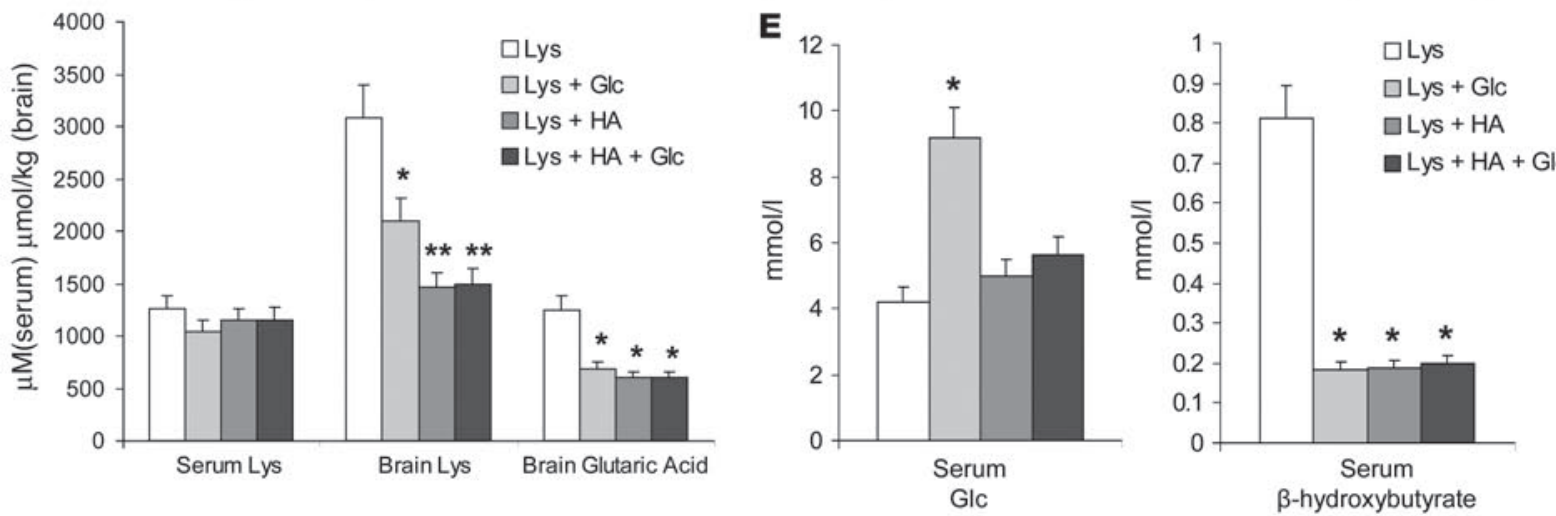

F

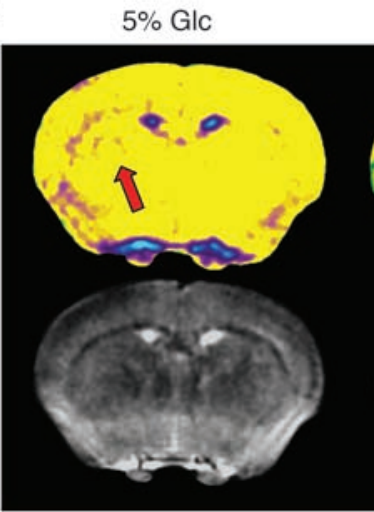

$5 \% \mathrm{HA}$

Glc

$\beta$-hydroxybutyrate

$5 \%$ Glc $+5 \%$ HA $\mathrm{T}_{2}[\mathrm{~ms}]$ $>80$

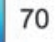

60 50 40 30 20 10 0

\section{Figure 7}

Glucose and homoarginine treatments increased survival and reduced brain injury in weanling Gcd $h^{-/-}$mice on the lysine diet. Twelve-day survival of weanling Gcdh $h^{-1-}$ mice on the lysine diet alone (black diamonds, $n=30$ ) or mice treated (black squares) with glucose $(\mathbf{A}, n=30$ ), homoarginine (HA) (B, $n=30)$, or combined glucose and homoarginine (C, $n=30)$. (D) Serum lysine, brain lysine, and brain glutaric acid levels and $(E)$ serum glucose and $\beta$-hydroxybutyrate levels in weanling $\mathrm{Gcdh}^{-1-}$ mice on the lysine diet with glucose, homoarginine, or both for 48 hours compared with lysine diet alone. Mean \pm SEM, ${ }^{*} P<0.01 ;{ }^{* \star} P<0.001 . n=6$ each group. (F) $\mathrm{T}_{2}$ maps (top) and $\mathrm{T}_{2}$-weighted images (bottom) of weanling $\mathrm{Gcdh}^{-/-}$mice with indicated treatments for 6 days show mild brain injury development in those treated with glucose alone (red arrow). Color bar indicates different $\mathrm{T}_{2}$ values (right side).

glutamine/creatine $(\mathrm{Glx} / \mathrm{Cr})$ ratio $(P<0.03)$ and the $\mathrm{GABA} / \mathrm{Cr}$ ratio $(P<0.05)$ but not the $\mathrm{N}$-acetyl aspartate (NAA)/Cr ratio was detectable by ${ }^{1} \mathrm{H}$ NMR spectroscopy after 48 hours of lysine diet exposure (Figure 9, A and B). These changes are consistent with our data from brain extracts (Figure 5D) and further show that glutamate and GABA depletion precede substantial neuronal damage, detected by a decrease in the NAA/Cr ratio as previously shown in human GA-I (28). Additionally, Glx/Cr and $\mathrm{GABA} / \mathrm{Cr}$ ratios were higher with homoarginine $(P<0.01)$ and combined treatment $(P<0.01)$ but not glucose alone (Figure $9 B)$. These data are consistent with glutamate and GABA levels from brain extracts in treated compared with untreated controls (Figure 8A) and suggest that ${ }^{1} \mathrm{H}$ NMR spectroscopy may be used to indicate impending brain injury.

\section{Discussion}

In this work we present several lines of evidence to suggest that the brain injury of GA-I involves mitochondrial disruption precipitated by glutaric acid production in the neuronal compartment from available lysine. Consistent with initial neuronal injury, expression of $G c d h$ and the first enzyme in lysine degradation, lysineoxoglutarate reductase (29), are both limited to neurons. These data implicate the neuronal compartment as the predominant location of lysine catabolism and glutaric acid production in the brain. Intracellular glutaric acid accumulation may cause direct mitochondrial toxicity within neurons. Previous studies with isolated mitochondria showed swelling induced with 500-1,000 $\mu \mathrm{M}$ glutaric acid (30). Higher concentrations of glutaric acid are consistently found in the brains of $\mathrm{Gcdh}^{-/-}$mice (12) and GA-I patients associated 

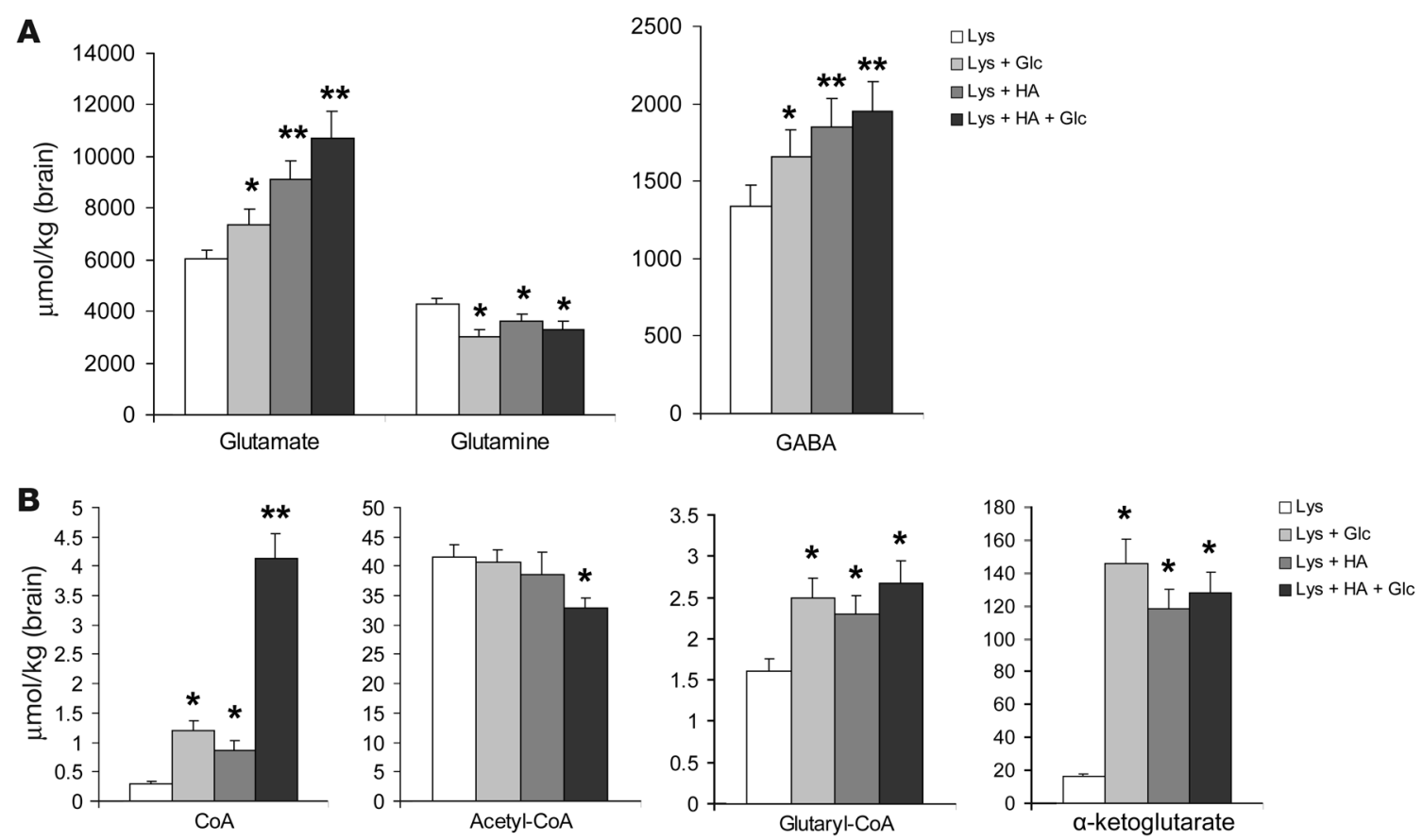

Figure 8

Glucose and homoarginine decrease biochemical changes associated with lysine diet exposure. (A) Glutamate, glutamine, and GABA levels with indicated treatments in weanling Gcdh ${ }^{-/}$mice compared with lysine diet alone at 48 hours. Mean $\pm \mathrm{SEM},{ }^{*} P<0.01 ;{ }^{* \star} P<0.001 . n=6$ each group. (B) Free CoA, acetyl-CoA, glutaryl-CoA, and $\alpha$-ketoglutarate levels in weanlings with indicated treatments compared with lysine diet alone. Mean \pm SEM. $n=6$ each group.

with injury (6). In the current study, mitochondrial swelling and biochemical changes consistent with Krebs cycle disruption were observed when brain glutaric acid levels reached more than 1,000 $\mu \mathrm{M}$. Glutaric acid is a preferred substrate for the mitochondrial oxodicarboxylate carrier that normally exchanges $\alpha$-ketoadipate for $\alpha$-ketoglutarate in a strict counter-exchange mechanism (31). The level of glutaric acid accumulation, depletion of $\alpha$-ketoglutarate, and accumulation of acetyl-CoA are all consistent with disruption of Krebs cycle function through loss of cycle intermediates as diagrammed in Figure 6.

Previous studies have shown a developmental change in BBB transport kinetics for basic and neutral amino acids that switch from low affinity/high capacity in the immature brain to high affinity/low capacity with maturity (15). Consistent with our current findings, this developmental difference was previously shown to provide a 3- to 4-fold decrease in brain lysine influx between suckling and adult rats (32). High-capacity amino acid uptake in the immature brain supports the increased metabolic demand and protein turnover during rapid growth and myelination of the brain. However, this enhanced influx of amino acids also provides agedependent susceptibility to GA-I and other metabolic disorders. The immature brain readily uses alternate energy substrates such as ketone bodies, which may be supplied to the brain as ketogenic amino acids (i.e., lysine and branched-chain amino acids). Ketogenic amino acids have been shown to provide a substantial proportion of ketone bodies used for myelin synthesis (33). As brain growth and myelination are completed, metabolic demands are reduced and the brain uses glucose more exclusively (15). Accordingly, transport and catabolic enzyme activity for lysine are reduced in the brain with maturity $(32,34)$. Therefore the immature brain is espe- cially susceptible to metabolic disorders of amino acid metabolism such as GA-I and maple syrup urine disease (35). This susceptibility is typically realized in the context of catabolic stress associated with fasting during a nonspecific illness. Catabolic stress leads to breakdown of muscle protein to free amino acids and enhanced amino acid uptake and turnover in the liver for gluconeogenesis (16). Hypoglycemia in children with GA-I during metabolic crisis (8) and in $\mathrm{Gcdh}^{-/-}$mice on a lysine diet suggests that the process of gluconeogenesis from available amino acids is impaired. Decreased glucose levels place greater dependence on ketone bodies and ketogenic amino acids in the brain. The combination of catabolic stress and enhanced amino acid access to the immature brain provides the opportunity for large accumulations of aberrant metabolites in GA-I and other disorders of amino acid metabolism. These agedependent susceptibilities, related to GA-I, and the proposed effect of treatments are diagrammed in Figure 10.

Recent evidence shows that glutaric acid has limited BBB permeability, suggesting de novo synthesis rather than diffusion or transport of glutaric acid into the brain (14). To confirm that glutaric acid is produced in the brain de novo from available lysine, we demonstrated that blocking brain lysine uptake with homoarginine decreased glutaric acid levels, resulting in reduced brain injury and increased survival. These findings are likely to have clinical relevance, since elevated brain glutaric acid levels and brain injury in GA-I patients occur despite the use of low lysine diets (4, 7). In the present study, controlling brain glutaric acid levels with homoarginine and glucose prevented encephalopathy, underscoring the importance of this strategy for treatment.

Currently, there is no reliable marker that can be used noninvasively for predicting the risk of brain injury in human GA-I. 
A ND Lys diet Lys diet + Glc + HA
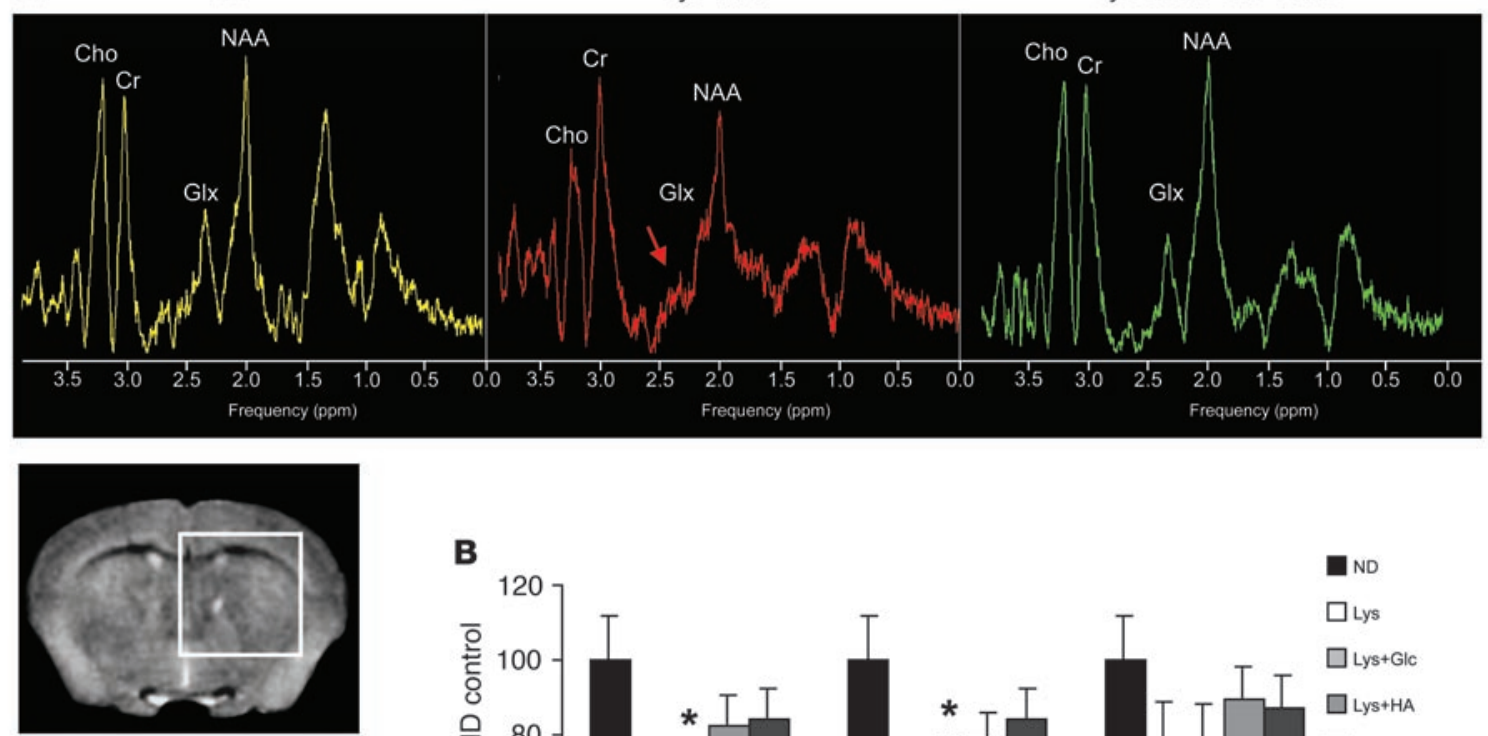

B
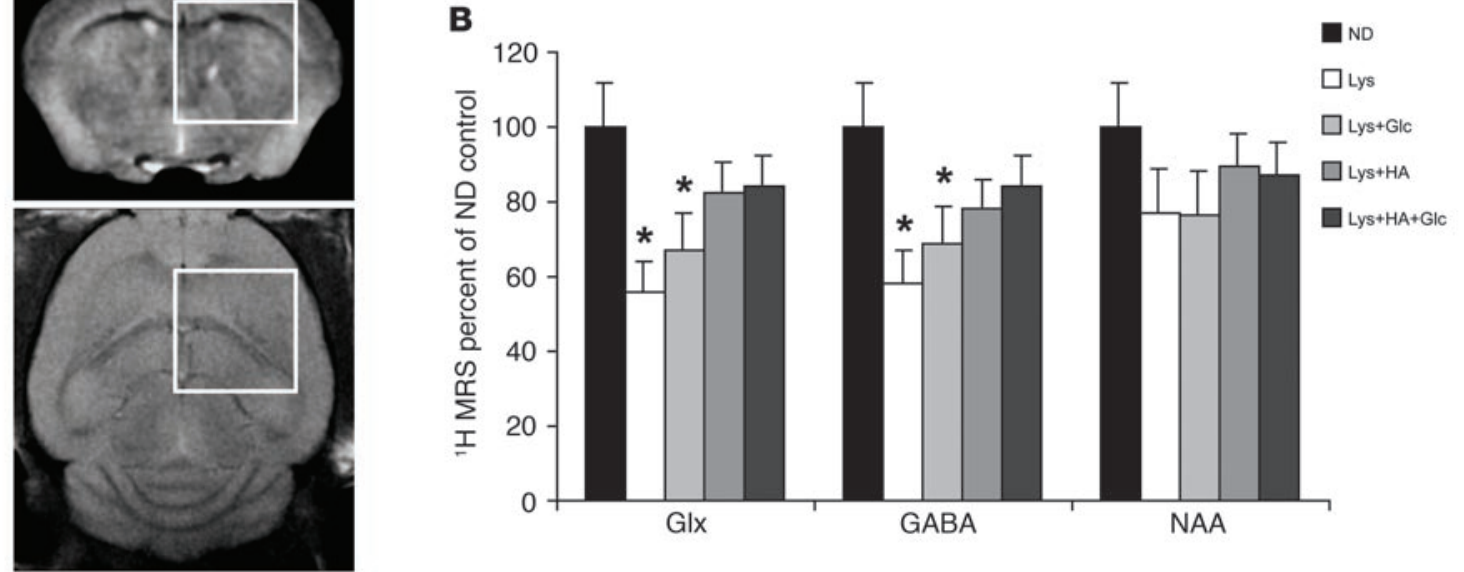

Figure 9

Glx and GABA depletion detected by ${ }^{1} \mathrm{H}$ NMR spectroscopy correlates with treatment success. (A) Comparison of ${ }^{1} \mathrm{H}$ NMR spectra from weanling $\mathrm{Gcdh}^{-1-}$ mice on a normal (left) or lysine diet (middle) or with combined glucose/homoarginine treatment (right) indicates a large decrease in the Glx peak (red arrow, center) and a smaller decrease in NAA after 48 hours of lysine diet exposure that was prevented by combined treatment (right). Multiple resonance peaks for GABA are not indicated. (B) $\mathrm{Glx} / \mathrm{Cr}$ ratio, $\mathrm{GABA} / \mathrm{Cr}$ ratio, and NAA/Cr ratio detected by ${ }^{1} \mathrm{H}$ NMR spectroscopy in weanling Gcdh $h^{-/}$mice on the lysine diet or with indicated treatments. Data are reported as percentage of normal diet control. Mean \pm SEM, ${ }^{*} P<0.03$. $n=4$ per group. Lower left panel indicates voxel location used to acquire NMR spectroscopy data.

Brain glutaric acid levels were previously shown to correlate with injury in this mouse model (12) but currently cannot be measured noninvasively. Glutamate and GABA depletion monitored by ${ }^{1} \mathrm{H}$ NMR spectroscopy correlates with increased brain glutaric acid levels and may translate for use in human GA-I to detect risk of brain injury. Glutamate depletion in this mouse model is consistent with reduced brain glucose utilization, as previously shown with immature rats (36). Reduced brain glucose utilization was previously shown in human GA-I using ${ }^{18}$ fluoro-2-deoxyglucose uptake studies (37), indicating that glutamate levels may also be compromised in human GA-I with encephalopathy. Glutamate is the precursor for GABA (38), and reduced GABA levels in this mouse model and human GA-I (7) correlate with glutamate depletion and further indicate that glutamate levels may be compromised in human GA-I. GABA levels may be initially reduced along with glutamate as brain glucose utilization is compromised during encephalopathy (37). Glutaric acid accumulation suppresses GABA production (21), and the return of glutamate levels with restored brain glucose utilization may set up unopposed excitatory neurotransmission resulting in seizures and excitotoxic lesions found in this model and in human GA-I (9). This possibility may explain the differences between glucose and homoarginine treatments and emphasizes the importance of controlling brain glutaric acid accumulation for neuroprotection.

Establishment of this mouse model provides the opportunity to test the effects of current and novel treatment strategies. Intravenous glucose administration is standard treatment for GA-I encephalopathy, but there are currently no data on the effect of this treatment in the brain. The lysine diet induces a catabolic state in $\mathrm{Gcdh}^{-/-}$mice similar to that seen in children with GA-I during crisis, including hypoglycemia and ketosis (Figure 4C). Catabolic stress leads to increased amino acid accumulation and breakdown in the liver (16). For lysine, increased catabolism was shown to be regulated by mitochondrial influx (39), and glucose availability reduces this effect $(39,40)$. We propose that a similar mechanism regulates lysine utilization in the immature brain. In the context of catabolic stress with reduced serum glucose levels, the immature brain depends on alternate energy substrates such as ketone bodies and ketogenic amino acids. Glucose supplementation provides adequate preferred substrate and reduces the demand for alternate substrates. This reduced demand correlates with reduced brain lysine accumulation in adult brains that 


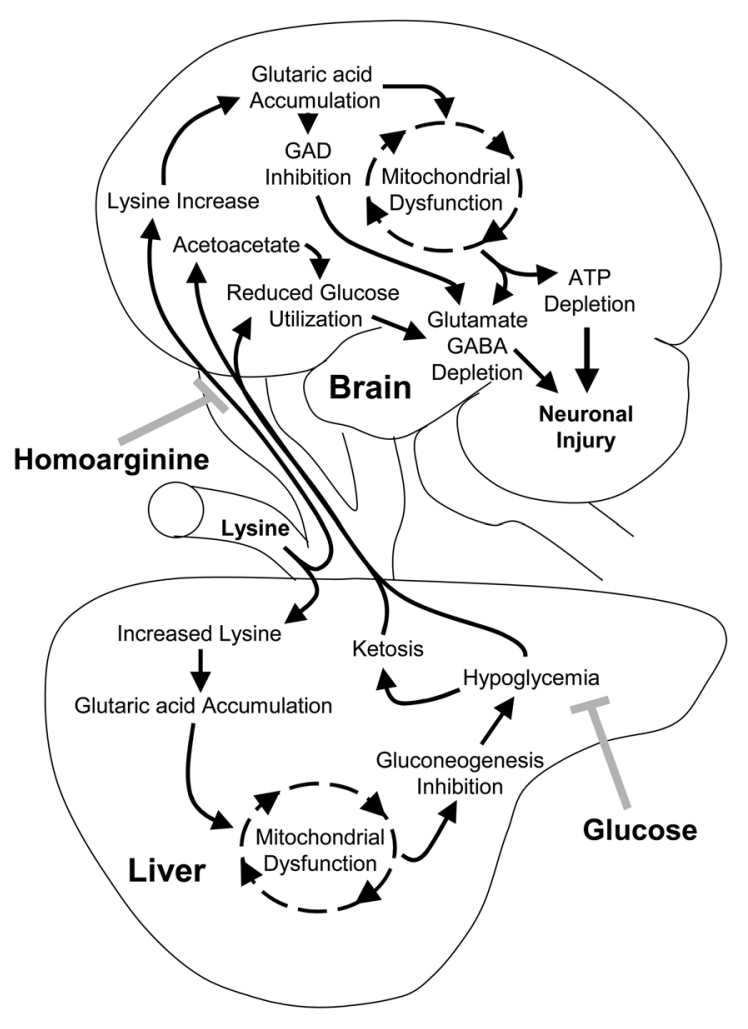

utilize glucose more exclusively (15). Here we demonstrate that dietary glucose therapy reduced lysine catabolism (glutaric acid formation) more than lysine accumulation in the brain. Serum glutaric acid levels were also lower with glucose treatment (data not shown), consistent with the influence of glucose to reduce amino acid turnover in the liver (41).

Combined homoarginine and glucose treatment may provide the best protection by reducing the substrate and the drive for glutaric acid production. However, the lack of cumulative effect on glutaric acid levels suggests that each treatment affects a different part of the same pathway. Glutaric acid is produced from lysine breakdown in the context of GCDH deficiency. The ratelimiting step of lysine breakdown at the mitochondrial level was shown to be lysine influx, which was increased by a high-protein diet and reduced by glucose availability (39). Our current data support a model in which lysine access is limited by homoarginine at the BBB and by glucose at the mitochondrial level. Both glucose and homoarginine reduced lysine breakdown (glutaric acid formation) similarly, but brain lysine accumulation remained higher with glucose treatment compared with homoarginine. Therefore, both treatments reduce brain glutaric acid production by limiting lysine access, although to different compartments.

Similar to Huntington disease, the expression of the affected gene in GA-I is not specific to the striatum but results in selective striatal degeneration. Medium spiny neurons are primarily affected in both disorders $(4,10)$. Although the clinical presentation of GA-I is commonly associated with acute onset, the resulting neuropathology in this mouse model is strikingly similar to that of Huntington disease, including the involvement of cortical pyramidal neurons $(12,42)$. Corticostriatal circuitry may play a role in the pathophysiology of both disorders (43). Further study

\section{Figure 10}

Proposed mechanism of susceptibility and effect of treatment. Increased lysine accumulation associated with the catabolic state of fasting illness or lysine loading results in accumulation of glutaric acid in the context of GCDH deficiency. Increased glutarate accumulation in the brain and liver leads to Krebs cycle inhibition as proposed in Figure 6. In the liver, Krebs cycle inhibition leads to inhibition of gluconeogenesis resulting in hypoglycemia. Hypoglycemia stimulates the use of alternate energy substrates in the brain including ketone bodies and ketogenic amino acids. Increased brain lysine utilization leads to enhanced glutarate accumulation and Krebs cycle inhibition. Krebs cycle inhibition in the brain results in depletion of glutamate, GABA, and ATP, with subsequent neuronal injury. Homoarginine blocks brain lysine uptake and controls glutarate accumulation from the substrate level. Glucose supplementation prevents hypoglycemia and the demand for ketone bodies and ketogenic amino acids.

of this mouse model may reveal factors underlying striatal-specific susceptibility that can be used to develop protective strategies.

The current findings provide insight into the age-dependent mechanism, treatment, and monitoring of GA-I and offer new strategies for the prediction and prevention of brain injury. Enhanced amino acid accumulation in the immature brain may contribute to the age-dependent susceptibility in other neurometabolic disorders (44). Competitive transport inhibition, shown in this study using homoarginine, provides an attractive approach to developing potential treatments for these disorders.

\section{Methods}

Materials. All chemicals were purchased from Sigma-Aldrich unless otherwise specified.

Animals. All animal experiments were approved by the Pennsylvania State University College of Medicine Institutional Animal Care and Use Committee. Gcdh ${ }^{-1-}$ mice and age-matched WT controls, both of mixed C57Bl/6J × 129SvEv background (45), were generated from homozygotes maintained at Penn State College of Medicine (Department of Comparative Medicine) in accordance with Institutional Animal Care and Use Committee research guidelines set forth by Pennsylvania State University and the Society for Neuroscience policy on the use of animals in neuroscience research. Heterozygotes $\left(\mathrm{Gcdh} h^{-/+}\right)$were generated by mating $\mathrm{Gcdh} h^{-/}$and WT mice. Gcd $b^{-/-}$ mice were previously produced by replacement of the first 7 exons of Gcdh with an in-frame $\beta$-gal cassette including a nuclear localization sequence. Therefore, $\mathrm{Gcdh}^{-/+}$and $\mathrm{Gcdh^{-/- }}$ mice express $\beta$-gal under the control of the $G c d h$ regulatory elements (45). To detect expression of $\beta$-gal, we used heterozygotes to avoid loss of cells that require GCDH during development.

Special diets. Diets were purchased from Harland Teklad. The normal diet was the Harland Teklad 2018 diet, which is $18 \%$ protein. The high-protein diet (TD.03637) (70\% casein) contained $62 \%$ protein, which is $4.7 \%$ lysine by weight. The lysine diet (TD.04412) was prepared by adding free lysine to a standard diet to achieve $4.7 \%$ total lysine. This level of lysine is not toxic in normal animals $(46,47)$. The lysine-homoarginine diet was prepared by adding $5 \%$ homoarginine to the lysine diet. Glucose was administered by adding $5 \%$ glucose monohydrate by weight to water and provided ad libitum. For intervention studies, male and female $\mathrm{Gcdh}^{-/-}$mice were placed on special diets at 4 weeks of age, housed 2 per cage, and observed every 12 hours; mice and diets were weighed daily ( $n=30$ for each study group). All special diet-treated animals and corresponding controls were evaluated daily for symptoms as previously described (12).

Neuropathology. To follow the earliest pathologic events, $12 \mathrm{Gcdh}^{-/-}, 5$ $\mathrm{Gcdh}^{-/+}$, and 5 WT mice were placed on a high-protein diet at 4 weeks of 
age. Two 4-week-old Gcdh ${ }^{-/-}, G c d h^{-/+}$, and WT mice were sacrificed before starting the diet ( 0 hour control), and 2 more $\mathrm{Gcdh}^{-/-}$mice were sacrificed every 12 hours after starting the diet and processed for electron microscopy. Three remaining Gcdh ${ }^{-/+}$and WT mice were sacrificed 60 hours after starting the diet and processed for electron microscopy. A second group of $12 \mathrm{Gcdh}^{-/-}, \mathrm{Gcdh^{-1+ }}$, and WT mice at 4 weeks of age were placed on the lysine diet. Three $G c d b^{-/}, G c d h^{-/+}$, and WT mice were sacrificed every 12 hours and processed for histology.

For histology, mice were anesthetized with $100 \mathrm{mg} / \mathrm{kg}$ pentobarbital (i.p.), perfused with lactated Ringers (Baxter) followed by $4 \%$ paraformaldehyde for 15 minutes. Brains were postfixed in $4 \%$ paraformaldehyde for 48 hours and paraffin embedded. For electron microscopy, mice were perfused with $4 \%$ paraformaldehyde with $1 \%$ glutaraldehyde in $0.2 \mathrm{M}$ cacodylate buffer. Brains were removed and postfixed for 48 hours and transferred to perfusion buffer until processed.

Histology. H\&E slides were prepared from paraffin-embedded brains. Sequential $10-\mu \mathrm{m}$ thick coronal brain slices were made within $0.5 \mathrm{~mm}$ of the bregma line for striatal sections and between bregma -1.5 and -2.0 for hippocampal sections (48).

Electron microscopy. Brains were dissected into coronal, hippocampal, or striatal blocks. Toluidine blue-stained semithin sections $1-\mu \mathrm{m}$ thick were made from these blocks. Ultrathin sections of $90 \mathrm{~nm}$ were then cut from the same blocks and stained with uranyl acetate and lead citrate for transmission electron microscopic analysis using a Philips CM10 transmission electron microscope.

Immunohistochemistry and confocal microscopy. GFAP, $\beta$-gal, neuron-specific nuclear protein (NeuN), neurofilament (N52), occludin (Occl), and choline acetyltransferase (ChaT) were detected using deparaffinized $10-\mu \mathrm{m}$-thick coronal brain slices. For $\beta$-gal colocalization, tissue was blocked with normal serum and incubated with monoclonal $\alpha$-GFAP (1:500) (SigmaAldrich), monoclonal $\alpha$-NeuN (1:20) (Millipore), or monoclonal $\alpha$-Occl (1-200) (Invitrogen) at the same time as with polyclonal $\alpha-\beta$-gal (1:200) (Millipore). Additional slides were incubated with polyclonal $\alpha$-GFAP (1:500) (Dako) and monoclonal $\alpha$-N52 (1-200) (Sigma-Aldrich) at the same time or polyclonal $\alpha$-ChaT (1:200) (Millipore) alone. All incubations were in PBS with normal serum overnight at $4^{\circ} \mathrm{C}$. The slides were washed separately 3 times each in PBS and incubated with goat anti-rabbit IgG coupled to $\mathrm{Cy}^{2}$ for $\beta$-gal and goat anti-mouse IgG coupled to $\mathrm{Cy}^{3}$ for NeuN, Occl, ChaT, or monoclonal GFAP. Polyclonal GFAP was detected with goat antirabbit coupled to $\mathrm{Cy}^{3}$, and N52 was detected with goat anti-mouse coupled to $\mathrm{Cy}^{2}$ (all secondary antibodies diluted 1:2,000; Jackson ImmunoResearch Laboratories Inc.). All slides were counterstained with DAPI, $0.1 \mu \mathrm{g} / \mathrm{ml}$ in PBS for 5 minutes. Confocal microscopy was performed using a Leica TCS SP2 AOBS confocal microscope (Leica Microsystems).

Biochemical analysis. $\mathrm{Gcdh}^{-/-}, \mathrm{Gcdb^{-/ }}$, and WT mice were placed on the normal or lysine diet at 4 and 8 weeks of age in groups of 6 ( 4 different groups by diet and age for each genotype). To detect differences in biochemical changes with intervention treatments, 4-week-old $\mathrm{Gcdh}^{-/-}$mice were placed on the lysine diet with $5 \%$ glucose added to the water $(n=6)$, or $5 \%$ homoarginine added to the diet $(n=6)$, or both $(n=6)$ for 48 hours or 2 weeks $(n=6)$. Mice were anesthetized with $100 \mathrm{mg} / \mathrm{kg}$ pentobarbital (i.p.). Serum and brains were collected and frozen to $-80^{\circ} \mathrm{C}$. Brains were extracted with $8 \%$ perchloric acid (PCA) and neutralized with potassium hydroxide as previously described (49). All neutralized PCA extracts and serum samples were kept at $-80^{\circ} \mathrm{C}$ until analysis.

Amino acids. Serum and brain amino acids were derivatized with phenyl isothiocyanate (PITC) and separated and quantified by reverse-phase highpressure liquid chromatography as previously described (50) using a $C_{18}$ Econosphere column $(150 \mathrm{~mm} \times 4.6 \mathrm{~mm}$ ID, $3 \mu \mathrm{m})$ from Alltech with norleucine $(100 \mu \mathrm{M})$ as an internal standard.
Glutaric acid and 3-OHGA. Serum samples and brain extracts were analyzed by gas chromatography-mass spectroscopy using stable-isotope dilution as previously described (51). Deuterium-labeled internal standards for GA (d4-GA) $(0.05 \mathrm{mg} / \mathrm{ml})$ and 3-OHGA (d5-HGA) $(0.05 \mathrm{mg} / \mathrm{ml})$ were added at $0.05 \mathrm{ml}$ each to $0.5 \mathrm{ml}$ serum samples or $1.5 \mathrm{ml}$ brain homogenates. Sulfosalicylic acid at $0.15 \mathrm{ml}$ of $9.33 \%$ or $70 \mathrm{mg}$ was added to serum or brain samples, respectively. Samples were extracted twice with $3 \mathrm{ml}$ diethyl ether and $1.5 \mathrm{ml}$ ethyl acetate. Combined organic phases were dried at $30^{\circ} \mathrm{C}$ under nitrogen and derivatized with $0.05 \mathrm{ml} \mathrm{BTSFA} / 1 \%$ TMCS [N,O-Bis(trimethylsilyl)trifluoroacetamide with trimethylchlorosilane] for 20 minutes at $80^{\circ} \mathrm{C}$. Injected volume of $0.001 \mathrm{ml}$ was analyzed using a Hewlett-Packard 6890 gas chromatograph with a 5973 mass spectrometer. The mass spectrometer monitored ions at 265/261 (with check of ratio at 237/233) for glutaric and 188/185 (check ratio at 262/259) for 3 -OHGA in separate runs.

ATP and phosphocreatine. Gcdh ${ }^{-/-}$and WT mice at 4 and 8 weeks of age were placed on the normal or lysine diet in groups of 4 ( 8 different groups by genotype, diet, and age). All mice were sacrificed by focused beam microwave irradiation to preserve phosphorylation status as previously described (52). The cortex, hippocampus, and striatum were dissected from both sides of the brain from each mouse and frozen. Samples from each brain area were weighed and extracted with $8 \%$ PCA as described above and neutralized. Neutralized PCA extracts were assayed for ATP and phosphocreatine enzymatically (49). ATP was measured by following the formation of NADPH spectrophotometrically using hexokinase coupled with glucose6-phosphate dehydrogenase in the presence of glucose and NADP+. Phosphocreatine was measured by adding $\mathrm{Cr}$ kinase to the above reaction and following the additional formation of NADPH. Protein concentrations were measured by the method of Lowry (53).

Coenzyme A, glutaryl-CoA and $\alpha$-ketoglutarate. PCA extracts of brain samples from each group described above were used to analyze free reduced CoA and glutaryl-CoA by HPLC as previously described (54). For $\alpha$-ketoglutarate, brain extracts were extracted with ethyl acetate. Dried residues were reconstituted in $25 \mathrm{mM}$ potassium phosphate buffer at $\mathrm{pH}$ 2.5. CoA, acetyl-CoA, glutaryl-CoA, and $\alpha$-ketoglutarate standards were used to develop a curve for quantification.

MRI and $H^{1}$ spectroscopy. MRI was performed on a $7.0 \mathrm{~T}$ Bruker system using a 2 -mm birdcage coil at 48 hours, 72 hours, and 6 days following the start of special diets ( $n=4$ mice per diet group). Mice were anesthetized with isoflurane (1-1.5\%) and imaged with a $\mathrm{T}_{2}$-weighted multiecho, spin echo sequence $(50.5 \mathrm{~mm}$ thick slices, $\mathrm{TR} / \mathrm{TE}=3,000 / 10.6-148.4 \mathrm{~ms}$, 14 echoes, $117 \times 117 \mu \mathrm{m}^{2}$ resolution, 2 averages; TR, repetition time; TE, echo time). $\mathrm{T}_{2}$ values were calculated on a pixel-by-pixel basis from the corresponding exponential fits using CCHIPS software (55). ${ }^{1} \mathrm{H}$ NMR spectroscopy data were acquired between 48 and 72 hours, using PRESS sequence $(\mathrm{TR} / \mathrm{TE}=2656 / 7 \mathrm{~ms}, 512$ averages, $4.2 \mathrm{ml}$ voxel covering the cortex and the striatum; Figure 6A). NMR spectroscopy data was processed using LCModel software (56). Data quality was assessed by visual inspection of the peaks in addition to quantitative measurement of the line width (full width as half maximum), automatically provided by LCModel software (56). Mean SD was greater than $20 \%$ for estimated concentration of reported metabolites. Mann-Whitney $U$ test was used for statistical analysis. Data are presented as a ratio to $\mathrm{Cr}$, with the assumption that its concentration was unaffected.

Statistics. Normally distributed data sets were analyzed by ANOVA with Fisher least-significant-difference post hoc test. Kruskal-Wallis 1-way ANOVA on ranks was performed with Student-Neuman-Keuls post hoc test on samples that were not normally distributed. SigmaStat software (Jandel Scientific) was used for analysis. All $P$ values of less than 0.05 were considered statistically significant. 


\section{Acknowledgments}

We thank Alistair Barber and Rhona Ellis of the Penn State Microscopy Imaging Core facility for their expertise in confocal microscopy and Javad Towfighi, Victor Canfield, and Cathleen McNeill for critical review of the manuscript. This work was supported by grants from the Laverty and Oxford Foundations, the Jake Gittlen Cancer Research Foundation, the Pennsylvania Keystone Initiative Award, the Life Sciences Greenhouse, the Tobacco Settlement Fund, and NIH grants 6R24RR017331 (to K.C. Cheng), RO1 NS38641 (to K. LaNoue), and 1F32NS058164-01 and 1F32NS058164-01A1 (to J. Lazovic). Additionally the authors wish to acknowledge support of the Gordon and Betty Moore Foundation to the Caltech Brain Imaging Center and NCRR U24 RR021760 to R.E. Jacobs.

Received for publication January 25, 2007, and accepted in revised form July 16, 2007.

Address correspondence to: William J. Zinnanti or Keith C. Cheng, Jake Gittlen Cancer Research Foundation, Penn State College of Medicine, 500 University Drive, Hershey, Pennsylvania 17033, USA. Phone: (717) 531-5635; Fax: (717) 531-5634; E-mail:wjz105@ psu.edu (W.J. Zinnanti); kcheng76@gmail.com (K.C. Cheng).
1. Kyllerman, M., and Steen, G. 1980. Glutaric aciduria. A "common" metabolic disorder? Arch. Fr. Pediatr. 37:279.

2. Kolker, S., et al. 2006. Natural history, outcome, and treatment efficacy in children and adults with glutaryl-CoA dehydrogenase deficiency. Pediatr. Res. 59:840-847.

3. Baric, I., et al. 1998. Diagnosis and management of glutaric aciduria type I. J. Inherit. Metab. Dis. 21:326-340.

4. Goodman, S.I., and Frerman, F.E. 1995. Organic acidemias due to defects in lysine oxidation: 2-ketoadipic academia and glutaric academia. In The metabolic and molecular bases of inherited disease. C. Scriver, A. Beudet, W. Sly, and D. Valle, editors. McGraw-Hill. New York, New York, USA. 2195-2204.

5. Strauss, K.A., Puffenberger, E.G., Robinson, D.L., and Morton, D.H. 2003. Type I glutaric aciduria, part 1: natural history of 77 patients. Am J. Med. Genet. C Semin. Med. Genet. 121:38-52.

6. Funk, C.B., et al. 2005. Neuropathological, biochemical and molecular findings in a glutaric acidemia type 1 cohort. Brain. 128:711-722.

7. Leibel, R.L., et al. 1980. Glutaric acidemia: a metabolic disorder causing progressive choreoathetosis. Neurology. 30:1163-1168.

8. Goodman, S.I., Norenberg, M.D., Shikes, R.H., Breslich, D.J., and Moe, P.G. 1977. Glutaric aciduria: biochemical and morphologic considerations. J. Pediatr. 90:746-750.

9. Soffer, D., et al. 1992. Striatal degeneration and spongy myelinopathy in glutaric acidemia. J. Neurol. Sci. 107:199-204.

10. Vonsattel, J.P., et al. 1985. Neuropathological classification of Huntington's disease. J. Neuropathol. Exp. Neurol. 44:559-577.

11. Strauss, K.A., and Morton, D.H. 2003. Type I glutaric aciduria, part 2: a model of acute striatal necrosis. Am. J. Med. Genet. C Semin. Med. Genet. 121:53-70.

12. Zinnanti, W.J., et al. 2006. A diet-induced mouse model for glutaric aciduria type I. Brain. 129:899-910.

13. Esrefoglu, M., Gepdiremen, A., and Kurus, M. 2003. Ultrastructural clues for glutamate-induced necrosis in parietal and cerebellar neurons. Fundam. Clin. Pharmacol. 17:341-347.

14. Sauer, S.W., et al. 2006. Intracerebral accumulation of glutaric and 3-hydroxyglutaric acids secondary to limited flux across the blood-brain barrier constitute a biochemical risk factor for neurodegeneration in glutaryl-CoA dehydrogenase deficiency. J. Neurochem. 97:899-910.

15. Cornford, E.M., and Cornford, M.E. 1986. Nutrient transport and the blood-brain barrier in developing animals. Fed. Proc. 45:2065-2072.

16. Daniel, P.M., Pratt, O.E., and Spargo, E. 1977. The mechanism by which glucagon induces the release of amino acids from muscle and its relevance to fasting. Proc. R. Soc. Lond. B Biol. Sci. 196:347-365.

17. Russell, R.R., 3rd, and Taegtmeyer, H. 1992. Coen- zyme A sequestration in rat hearts oxidizing ketone bodies. J. Clin. Invest. 89:968-973.

18. Yudkoff, M., Daikhin, Y., Nissim, I., Lazarow, A., and Nissim, I. 2001. Brain amino acid metabolism and ketosis. J. Neurosci. Res. 66:272-281.

19. Sweatt, A.J., Garcia-Espinosa, M.A., Wallin, R., and Hutson, S.M. 2004. Branched-chain amino acids and neurotransmitter metabolism: expression of cytosolic branched-chain aminotransferase (BCATc) in the cerebellum and hippocampus. J. Comp. Neurol. 477:360-370.

20. Colon, A.D., Plaitakis, A., Perakis, A., Berl, S., and Clarke, D.D. 1986. Purification and characterization of a soluble and a particulate glutamate dehydrogenase from rat brain. J. Neurochem. 46:1811-1819.

21. Stokke, O., Goodman, S.I., and Moe, P.G. 1976. Inhibition of brain glutamate decarboxylase by glutarate, glutaconate, and beta-hydroxyglutarate: explanation of the symptoms in glutaric aciduria? Clin. Chim. Acta. 66:411-415.

22. Schousboe, A., et al. 1997. Trafficking between glia and neurons of TCA cycle intermediates and related metabolites. Glia. 21:99-105.

23. Tews, J.K., and Harper, A.E. 1983. Atypical amino acids inhibit histidine, valine, or lysine transport into rat brain. Am. J. Physiol. 245:R556-R563.

24. Lazovic, J. 2005. Neuroinflammation and both cytotoxic and vasogenic edema are reduced in interleukin-1 type 1 receptor-deficient mice conferring neuroprotection. Stroke. 36:2226-2231.

25. Juliet, P.A., Balasubramaniam, D., Balasubramaniam, N., and Panneerselvam, C. 2003. Carnitine: a neuromodulator in aged rats. J. Gerontol. A Biol. Sci. Med. Sci. 58:970-974.

26. Kido, Y., et al. 2001. Functional relevance of carnitine transporter OCTN2 to brain distribution of L-carnitine and acetyl-L-carnitine across the bloodbrain barrier. J. Neurochem. 79:959-969.

27. Strauss, K.A. 2005. Glutaric aciduria type 1: a clinician's view of progress. Brain. 128:697-699.

28. Oguz, K.K., Ozturk, A., and Cila, A. 2005. Diffusion-weighted MR imaging and MR spectroscopy in glutaric aciduria type 1. Neuroradiology. 47:229-234.

29. Papes, F., Surpili, M.J., Langone, F., Trigo, J.R., and Arruda, P. 2001. The essential amino acid lysine acts as precursor of glutamate in the mammalian central nervous system. FEBS Lett. 488:34-38.

30. Palmeira, C.M., Rana, M.I., Frederick, C.B., and Wallace, K.B. 2000. Induction of the mitochondrial permeability transition in vitro by short-chain carboxylic acids. Biochem. Biophys. Res. Commun. 272:431-435

31. Fiermonte, G., et al. 2001. Identification of the human mitochondrial oxodicarboxylate carrier. Bacterial expression, reconstitution, functional characterization, tissue distribution, and chromosomal location. J. Biol. Chem. 276:8225-8230.

32. Banos, G., Daniel, P.M., and Pratt, O.E. 1978. The effect of age upon the entry of some amino acids into the brain, and their incorporation into cerebral protein. Dev. Med. Child Neurol. 20:335-346.
33. Dhopeshwarkar, G.A., and Subramanian, C. 1979. Lipogenesis in the developing brain: utilization of radioactive leucine, isoleucine, octanoic acid and beta-hydroxybutyric acid. Lipids. 14:47-51.

34. Rao, V.V., Pan, X., and Chang, Y.F. 1992. Developmental changes of L-lysine-ketoglutarate reductase in rat brain and liver. Comp. Biochem. Physiol. B. 103:221-224.

35. Morton, D.H., Strauss, K.A., Robinson, D.L., Puffenberger, E.G., and Kelley, R.I. 2002. Diagnosis and treatment of maple syrup disease: a study of 36 patients. Pediatrics. 109:999-1008.

36. Miller, A.L., Kiney, C.A., and Staton, D.M. 1984. Effects of lactate on glucose metabolism of developing rat brain. Brain Res. 316:33-40.

37. Al-Essa, M., et al. 1998. Fluoro-2-deoxyglucose (18FDG) PET scan of the brain in glutaric aciduria type 1: clinical and MRI correlations. Brain Dev. 20:295-301.

38. Shatunova, N.F., and Sytinsky, I.A. 1964. On the intracellular localization of glutamate decarboxylase and gamma-aminobutyric acid in mammalian brain. J. Neurochem. 11:701-708.

39. Blemings, K.P., Crenshaw, T.D., and Benevenga, N.J. 1998. Mitochondrial lysine uptake limits hepatic lysine oxidation in rats fed diets containing 5, 20 or $60 \%$ casein. J. Nutr. 128:2427-2434.

40. Daniel, P.M. 1977. The metabolic homoeostatic role of muscle and its function as a store of protein. Lancet. 2:446-448.

41. Hayashi, M., Konishi, K., Kameoka, A., and Mino, M. 1981. Serum free amino acid depletion in ketotic children. J. Nutr. Sci. Vitaminol. (Tokyo). 27:449-454.

42. Cudkowicz, M., and Kowall, N.W. 1990. Degeneration of pyramidal projection neurons in Huntington's disease cortex. Ann. Neurol. 27:200-204.

43. Cepeda, C., Wu, N., Andre, V.M., Cummings, D.M., and Levine, M.S. 2007. The corticostriatal pathway in Huntington's disease. Prog. Neurobiol. 81:253-271.

44. Filiano, J.J. 2006. Neurometabolic diseases in the newborn. Clin. Perinatol. 33:411-479.

45. Koeller, D.M., et al. 2002. Biochemical, pathologic and behavioral analysis of a mouse model of glutaric acidemia type I. Hum. Mol. Genet. 11:347-357.

46. Benevenga, N.J., and Steele, R.D. 1984. Adverse effects of excessive consumption of amino acids. Annu. Rev. Nutr. 4:157-181.

47. Tsubuku, S., Mochizuki, M., Mawatari, K., Smriga, M., and Kimura, T. 2004. Thirteen-week oral toxicity study of L-lysine hydrochloride in rats. Int. J. Toxicol. 23:113-118.

48. Franklin, K.B.J., and Paxinos, G. 1997. The mouse brain: in stereotaxic coordinates. Academic Press Inc. San Diego, California, USA. 216 pp.

49. Williamson, J.R., and Corkey, B.E. 1979. Assay of citric acid cycle intermediates and related compounds - update with tissue metabolite levels and intracellular distribution. Methods Enzymol. 55:200-222.

50. Hariharan, M., Naga, S., and VanNoord, T. 1993. Systematic approach to the development of plasma 
amino acid analysis by high-performance liquid chromatography with ultraviolet detection with precolumn derivatization using phenyl isothiocyanate. J. Chromatogr. 621:15-22.

51. Kolker, S., et al. 2003. Glutaryl-CoA dehydrogenase deficiency: region-specific analysis of organic acids and acylcarnitines in post mortem brain predicts vulnerability of the putamen. Neuropediatrics. 34:253-260.

52. O'Callaghan, J.P., and Sriram, K. 2004. Focused microwave irradiation of the brain preserves in vivo protein phosphorylation: comparison with other methods of sacrifice and analysis of multiple phosphoproteins. J. Neurosi. Methods. 135:159-168.

53. Lowry, O.H., Passonneau, J.V., Hasselberger, F.X., and Schulz, D.W. 1964. Effect of ischemia on known substrates and cofactors of the glycolytic pathway in brain. J. Biol. Chem. 239:18-30.

54. DeBuysere, M.S., and Olson, M.S. 1983. The analysis of acyl-coenzyme A derivatives by reverse-phase high-performance liquid chromatography. Anal. Biochem. 133:373-379.

55. Schmithorst, V.J., Dardzinski, B.J., and Holland, S.K. 2001. Simultaneous correction of ghost and geometric distortion artifacts in EPI using a multiecho reference scan. IEEE Trans. Med. Imaging. 20:535-539.

56. Provencher, S.W. 2001. Automatic quantitation of localized in vivo $1 \mathrm{H}$ spectra with LCModel. NMR Biomed. 14:260-264. 